\title{
Stereodynamics of Diatom Formation through
}

\section{Eley-Rideal Abstraction}

O. Galparsoro, ${ }^{*, \dagger, \dagger}$ J. I. Juaristi,,$^{\S, \|, \dagger}$ C. Crespos,,$+\uparrow$ M. Alducin, ${ }^{\|, \dagger}$ and P. Larrégaray

Donostia International Physics Center (DIPC), Paseo Manuel de Lardizabal 4, 20018 Donostia-San Sebastián, Spain, Université de Bordeaux, ISM, UMR 5255, F-33400 Talence, France, CNRS, ISM, UMR5255, F-33400 Talence, France, Departamento de

Física de Materiales, Facultad de Químicas (UPV/EHU), Apartado 1072, 20080

Donostia-San Sebastián, Spain, and Centro de Física de Materiales CFM/MPC (CSIC-UPV/EHU), Paseo Manuel de Lardizabal 5, 20018 Donostia-San Sebastián, Spain

E-mail: o.galparsoro@gmail.com

${ }^{*}$ To whom correspondence should be addressed

$\dagger$ Donostia International Physics Center (DIPC), Paseo Manuel de Lardizabal 4, 20018 Donostia-San Sebastián, Spain

$\ddagger$ Université de Bordeaux, ISM, UMR 5255, F-33400 Talence, France

ฯ CNRS, ISM, UMR5255, F-33400 Talence, France

$\S$ Departamento de Física de Materiales, Facultad de Químicas (UPV/EHU), Apartado 1072, 20080 Donostia-San Sebastián, Spain

"I Centro de Física de Materiales CFM/MPC (CSIC-UPV/EHU), Paseo Manuel de Lardizabal 5, 20018 Donostia-San Sebastián, Spain 


\begin{abstract}
Using adiabatic and nonadiabatic quasiclassical molecular dynamics simulations within the single adsorbate limit, we study the dependence on the incidence angle of the Eley-Rideal (ER) recombination of $\mathrm{H}_{2}$ and $\mathrm{N}_{2}$ on the (100) and (110) tungsten surfaces. In the nonadiabatic simulations, effects due to electron-hole pair excitations and the energy exchange between the recombination partners and the surface lattice are included in the dynamics by combining the local density friction approximation and the generalized Langevin oscillator model. Our adiabatic and nonadiabatic results, which are qualitatively similar, show that the incidence angle affects much more $\mathrm{N}_{2}$ recombination than $\mathrm{H}_{2}$ recombination due mainly to the higher corrugation of the potential energy surface for the former case. In particular, for $\mathrm{N}$ abstraction from W(110) we find a very efficient ER recombination pathway at grazing incidence angles. This new mechanism significantly influences the energy partition among the different degrees of freedom of the outgoing molecules. We also find that the energy losses only depend slightly on the incidence geometry and that, as in normal incidence, are dominated by phonons in the case of $\mathrm{N}_{2}$ recombination and by electron-hole pair excitations in the case of $\mathrm{H}_{2}$ recombination.
\end{abstract}




\section{Introduction}

Understanding the mechanisms of gas-surface elementary reactions is a critical issue in order to model surface chemistry of primary interest in topics such as oxidation, ${ }^{1}$ corrosion, ${ }^{2}$ hydrogen storage ${ }^{3}$ heterogeneous catalysis, ${ }^{4-6}$ plasma wall interactions, ${ }^{7,8}$ and chemistry of atmospheric $^{9,10}$ and interstellar media. ${ }^{11,12}$ One gas-surface elementary process that has received special attention in the last decades is diatom recombination via gas-phase atom scattering off adsorbates. Numerous theoretical ${ }^{13-41}$ and experimental studies ${ }^{42-58}$ have concerned recombination on metals. As atom chemisorption energies are usually large on such materials, thermal atom-atom recombination via the Langmuir-Hinshelwood reaction ${ }^{59}$ is ineffective at low temperatures. Rather, recombinations may proceed via Eley-Rideal (ER) or Hot-Atom (HA) abstraction. ${ }^{60,61}$ The recombinative abstraction of light projectiles (H,D), ${ }^{13,14,23,24,34,39,42,43,45,47,51,55}$ is considered to mainly proceed via the HA mechanism, in which the incoming gas-phase species experiences few collisions with the surface prior recombining with the adsorbate. Conversely, the ER abstraction, in which the impinging atom collides in the close vicinity of the adsorbate, is thought to be less efficient, with some exceptions such as $\mathrm{N}_{2}$ formation on $\mathrm{Ag}(111)^{35,53}$ and $\mathrm{H}$ recombination with adsorbed $\mathrm{Cl}$ on $\mathrm{Au}(111) .{ }^{23}$ However, this assertion should be considered with care since recent theoretical studies evidence that the ER and HA mechanisms may compete in the recombination of $\mathrm{H}_{2}$ from H-covered $\mathrm{W}(110)$ as a result of energy dissipation into electron-hole $(e-h)$ pair excitations and other adsorbates during the hyperthermal diffusion of the hot species. ${ }^{40,62}$ Thus, though the single-collision ER reaction cross section is generally small, its contribution to the total abstraction cross section might be significant.

As far as ER abstraction is concerned, theoretical studies have mainly scrutinized the dynamics at normal incidence. Different behaviors have been observed that depend on the recombining species and the metal surface under study. Energy dissipation to metal phonons is found to scale with the mass ratio between the incident species and the surface atom. ${ }^{16,37,38,41,50,52,63-65}$ In contrast, energy dissipation via $e$ - $h$ pair excitations is shown to 
be more important for $\mathrm{H}_{2}$ recombination than for $\mathrm{N}_{2}$ recombination on the $\mathrm{W}(100)$ and W(110) surfaces. ${ }^{66}$ Nevertheless, in both cases the effect of $e$ - $h$ pair excitations on the ER cross sections is found to be minor. The weak surface temperature effects observed for light atoms, such as $\mathrm{H}$ and $\mathrm{D},{ }^{41,42,50,63,65}$ turn to be more sizable as the mass of the recombining atoms/molecules increases. ${ }^{37,38,66}$ For recombinations involving hydrogen, isotopic substitutions have also been investigated in many of the systems mentioned above. ${ }^{50,51,67-69}$ In these studies, isotopic effects are observed for ER reactivity as well as for the internal energy distributions.

Since ER abstraction is ultrafast (subpicosecond time scale) and involves only one rebound of the projectile on the surface, it might be reasonable to think that the surface will act as a spectator and, consequently, plays a minor role in this kind of process. This is definitely not the case as significant crystallographic anisotropies have already been observed, in particular for $\mathrm{N}_{2}$ recombination on $\mathrm{W}(100)$ and $\mathrm{W}(110)$ for which the different corrugation of the potential energy surface (PES) is clearly an important ingredient ruling the dynamics. ${ }^{38}$ As a consequence of such a corrugation, stereodynamical effects, i.e., sensitivity of the recombination dynamics on the initial incidence angle of the gas phase partners, might be anticipated. This issue has been much less theoretically ${ }^{20,52,70}$ and experimentally ${ }^{44,71,72}$ studied. These few existing studies show already that the influence of the incidence angle depends much on the properties of the system. While the beam incidence does not lead to any sizable change of the recombination rate constants on $\mathrm{H}(\mathrm{D})$ atom reaction with adsorbed $\mathrm{D}(\mathrm{H})$ on $\mathrm{Pt}(111),{ }^{44}$ it is shown to greatly influence the $\mathrm{CO}$ oxidation by atomic oxygen on $\mathrm{Pt}(111), \operatorname{Ir}(111)$, and $\mathrm{Ru}(0001) \cdot{ }^{71}$ Besides, the outgoing angles of the NO molecules formed from the oxidation of $\mathrm{N}$ on the $\mathrm{O}$-covered $\mathrm{Ru}(0001)$ surface are shown to negligibly depend on the incidence angle. For $\mathrm{H}_{2}$ recombination on $\mathrm{Cu}(111)^{20,70}$ and $\mathrm{CO}$ oxidation on O-covered $\mathrm{Pt}(111),{ }^{52}$ the incidence angle slightly affects the reactivity itself, but significantly influences the final energy distributions of the formed molecules.

In this work, we analyze the ER recombination of $\mathrm{H}_{2}$ and $\mathrm{N}_{2}$ on the $\mathrm{W}(100)$ and $\mathrm{W}(110)$ 
surfaces at various incidence angles for a wide range of initial collision energies of the projectile $\left(\mathrm{E}_{i}=0.1-5.0 \mathrm{eV}\right)$. Although many studies have analized $\mathrm{H}+\mathrm{H}$ and $\mathrm{N}+\mathrm{N}$ ER reactions on $\mathrm{W},{ }^{21,27,36-38,40,66,68,73-80}$ to our knowledge none of them have focussed on the effect of the incidence angle of the projectile. This issue is here studied in detail putting special emphasis on understanding how the incidence angle affects the ER reaction paths, the energy dissipation to the metallic surface, and the final energy distribution of the formed molecules. The strong surface anisotropy was already observed for $\mathrm{N}_{2}$ recombination at normal incidence and the different roles that $e-h$ pairs and phonons have on these systems make them particularly appealing to this analysis. The organization of the paper is as follows. Section 2 gives details of the PESs and the quasiclassical trajectory (QCT) simulation methodology. Results from the dynamics simulations are discussed in section 3. Section 4 summarizes the main conclusions.

\section{Theoretical methods}

Off-normal incidence scattering of atomic $\mathrm{H}$ and $\mathrm{N}$ off, respectively, the $\mathrm{H}$ - and $\mathrm{N}$-preadsorbed $\mathrm{W}(100)$ and W(110) surfaces is investigated within the zero coverage limit (single adsorbate). QCT is used that relies on global six-dimensional (6D) PESs built by fitting density functional theory (DFT) energies with the flexible periodic London-Eyring-PolanyiSato (FPLEPS) model. ${ }^{81-83}$ By QCT we mean here that the zero point energy (ZPE) of the adsorbates is included in the initial conditions, although the subsequent propagation is treated classically. Details about the DFT calculations based on the PW91 exchangecorrelation functional $^{84}$ and the FPLEPS fitting procedure can be found in refs 79,85 for $\mathrm{H}+\mathrm{H} / \mathrm{W}(100)$ and $\mathrm{H}+\mathrm{H} / \mathrm{W}(110)$, in refs $82,83,86,87$ for $\mathrm{N}+\mathrm{N} / \mathrm{W}(100)$, and in refs $82,87-89$ for $\mathrm{N}+\mathrm{N} / \mathrm{W}(110)$. The methodology for molecular dynamics simulation at normal incidence has been detailed elsewhere ${ }^{66}$ so that only the main lines are recalled here.

In order to rationalize nonadiabatic effects in the abstraction process, simulations are 
performed within two different schemes. In the first one, we use as in refs $36,37,41$ the BornOppenheimer static surface approximation (BOSS), in which neither energy exchange with the surface phonons nor electronic excitations are accounted for. In the second one, we follow the LDFA-GLO scheme of ref 90 that permits to introduce in the dynamics both the effect of $e-h$ pair excitations and energy exchange to the surface lattice, while still using the adiabatic 6D PES. Specifically, energy exchange with the metal lattice is introduced through a threedimensional (3D) surface oscillator that is connected to a second 3D oscillator that acts as the thermal bath (generalized Langevin oscillator model, GLO ${ }^{91-95}$ ). We note in passing that the validity of the GLO model in describing the energy-exchange with the surface lattice under off-normal incidence conditions has been confirmed in ref 96 . The authors find that the GLO dissociative and scattering dynamics of $\mathrm{N}_{2}$ on $\mathrm{W}(110)$ is essentially similar to the results obtained from ab initio molecular simulations that also allow for the surface atoms movement. On the other hand, electronic nonadiabaticity is introduced by means of a dissipative (friction) force acting on the recombining atoms. This friction force is calculated within the local density friction approximation (LDFA). ${ }^{97}$ More details on the LDFA-GLO implementation for our specific systems can be found in ref 66 . 

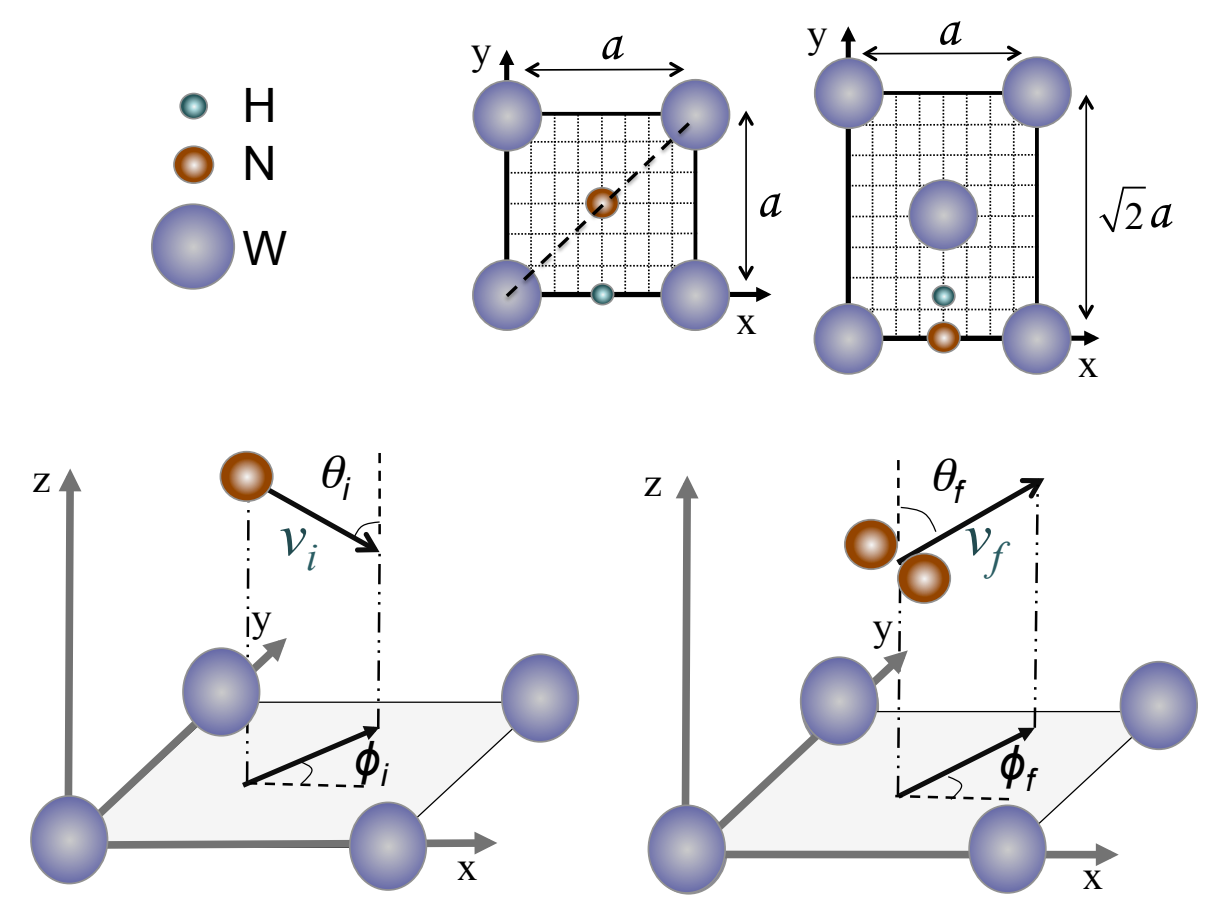

Figure 1: Up: adsorption sites for $\mathrm{H}$ and $\mathrm{N}$ atoms on $\mathrm{W}(100)$ (left) and $\mathrm{W}(110)$ (right). W atoms are in purple, $\mathrm{H}$ atoms are in blue and $\mathrm{N}$ atoms are in red. Down: Coordinate system defining the incidence angles and cartesian coordinates of the impinging atom (left) and the outgoing formed molecule (right).

All trajectories are integrated with a Beeman algorithm using a fixed time step of 0.01 fs, which results in an average energy conservation in the BOSS simulations of $0.003 \mathrm{meV}$, $0.008 \mathrm{meV}, 0.006 \mathrm{meV}$, and $0.028 \mathrm{meV}$ for $\mathrm{H}+\mathrm{H} / \mathrm{W}(100), \mathrm{H}+\mathrm{H} / \mathrm{W}(110), \mathrm{N}+\mathrm{N} / \mathrm{W}(100)$, and $\mathrm{N}+\mathrm{N} / \mathrm{W}(110)$, respectively. The initial conditions for the QCT simulations are the following. The adsorbed atom (target) is initially located at the most favorable adsorption site with velocity and position consistent with its ZPE, which is computed through a $x$, $y$, and $z$ mode decomposition. ${ }^{66}$ The adsorption sites for $\mathrm{H}$ and $\mathrm{N}$ on both $\mathrm{W}$ surfaces are illustrated in Figure 1 (up). The ZPEs and adsorption sites positions are those of ref 66. In order to conserve the ZPE of the target prior colliding with the incoming atom in the LDFA-GLO simulations, the friction force starts acting when the energy of the target exceeds the ZPE. This occurs when the target starts to interact with the projectile. The friction force is then applied until the end of the trajectory. Note that if we had not proceeded in 
that way, the ZPE would not have been conserved before the interaction with the incoming projectile due to the energy loss generated by the friction force. This would not have been solved by including a random force in the simulations linked to the friction force via the second fluctuation-dissipation theorem. Within this procedure, the thermal energy of the adsorbates before the interaction would be that corresponding to the temperature of the surface $(300 \mathrm{~K})$, which is much lower than the ZPE. The impinging gas-phase $\mathrm{H}(\mathrm{N})$ atom starts at $Z_{p}=7.0 \AA(8.0 \AA)$, i.e., in the asymptotic region of the potential, with initial collision energies $\mathrm{E}_{i}$ that vary within the range $0.1-5.0 \mathrm{eV}$. The direction of the projectile initial velocity $\mathbf{v}_{i}$ is defined by the polar angle with respect to the surface normal $\theta_{i}$ and the azimuthal angle with respect to the $x$-axis $\phi_{i}$. Similarly, $\theta_{f}$ and $\phi_{f}$ are the corresponding polar and azimuthal angles describing the direction of the final velocity of the center of mass $(\mathrm{CM})$ of the formed diatom $\mathbf{v}_{f}$. The coordinate system is depicted in Figure 1 (down). The incidence angles used in the present work for each system are as follows. Simulations of $\mathrm{H}_{2}$ recombination on $\mathrm{W}(100)$ are performed for $\theta_{i}=40^{\circ}$ and $80^{\circ}$ with $\phi_{i}=0^{\circ}, 45^{\circ}$, and $90^{\circ}$. Taking advantage of the $C_{4 v}$ symmetry for $\mathrm{N}$ abstraction on $\mathrm{W}(100), \theta_{i}=40^{\circ}$ and $80^{\circ}$ with only $\phi_{i}=0^{\circ}$ and $45^{\circ}$ are considered. Finally, for $\mathrm{H}_{2}$ and $\mathrm{N}_{2}$ recombination on $\mathrm{W}(110), \theta_{i}=40^{\circ}$ and $80^{\circ}$ with $\phi_{i}=0^{\circ}, 54.74^{\circ}$, and $90^{\circ}$ are simulated. For each incidence condition and system, the initial coordinates of the projectiles $\left(X_{p}, Y_{p}\right)$ are randomly sampled within an area that has been carefully selected in accordance to the specific symmetry of the system and that ensures projectile rebounds in the vicinity of the target. The size of each sampling area was gradually increased until the corresponding ER cross sections were converged. In all cases, a sampling density of 8444 trajectories $/ \AA^{2}$ was used to ensure good statistics.

The possible exit channels for the dynamics simulation are defined in detail elsewhere. ${ }^{36}$ Here we focus on the recombinative abstraction process that takes place whenever both atoms reach the initial altitude of the projectile with positive diatom CM momentum along the surface normal ( $z$-axis) and interatomic distance $r<2.2(2.5) \AA$ in $\mathrm{H}_{2}\left(\mathrm{~N}_{2}\right)$ recombination. Among the possible recombination events, the ER abstraction (recombination) process is 
assumed to occur when the formed molecule moves definitively towards the vacuum before the second rebound of the projectile.

The ER cross section $\sigma_{E R}$ is computed as

$$
\sigma_{E R}=\iint_{D} P\left(X_{p}, Y_{p}\right) d X_{p} d Y_{p}
$$

where the integration area $D$ is the sampling area. The two-dimensional (2D) opacity function $P\left(X_{p}, Y_{p}\right)$ is the probability of ER recombination for a given set of $X_{p}$ and $Y_{p}$ values.

\section{Results}

Qualitatively, the ER recombination dynamics obtained for each system from the BOSS and LDFA-GLO calculations exhibit the same general features. For this reason we use the BOSS results in section 3.1 to analyze in detail how the incidence angle affects different properties of the recombination dynamics and how these effects depend much on the recombining atoms and crystal face. The results from the LDFA-GLO calculations are discussed in section 3.2 putting special emphasis on the dependence of the energy loss channels ( $e-h$ pairs and phonons) on the incidence angle conditions.

\subsection{BOSS results: Eley-Rideal dynamics at off-normal incidence}

The BOSS ER cross sections $\sigma_{E R}$ for $\mathrm{N}_{2}$ recombination from $\mathrm{W}(100)$ at different incidence angles $\left(\theta_{i}, \phi_{i}\right)$ are displayed in Figure 2 as a function of the projectile collision energy $\mathrm{E}_{i}$. The effect of the incidence angle in $\mathrm{N}+\mathrm{N} / \mathrm{W}(100)$ is particularly significant at low collision energies. ER abstraction at normal incidence in this system was previously found to involve a collision energy threshold of $0.53 \mathrm{eV}$, despite the existence of nonactivated recombination pathways. ${ }^{36,38}$ Such a threshold was attributed to a dynamical effect resulting from an

interplay between the $\mathrm{N}-\mathrm{N}$ repulsion at the entrance channel and the strong attraction of the projectile towards the top W surface atoms. Figure 2 (left panel) shows that the nor- 

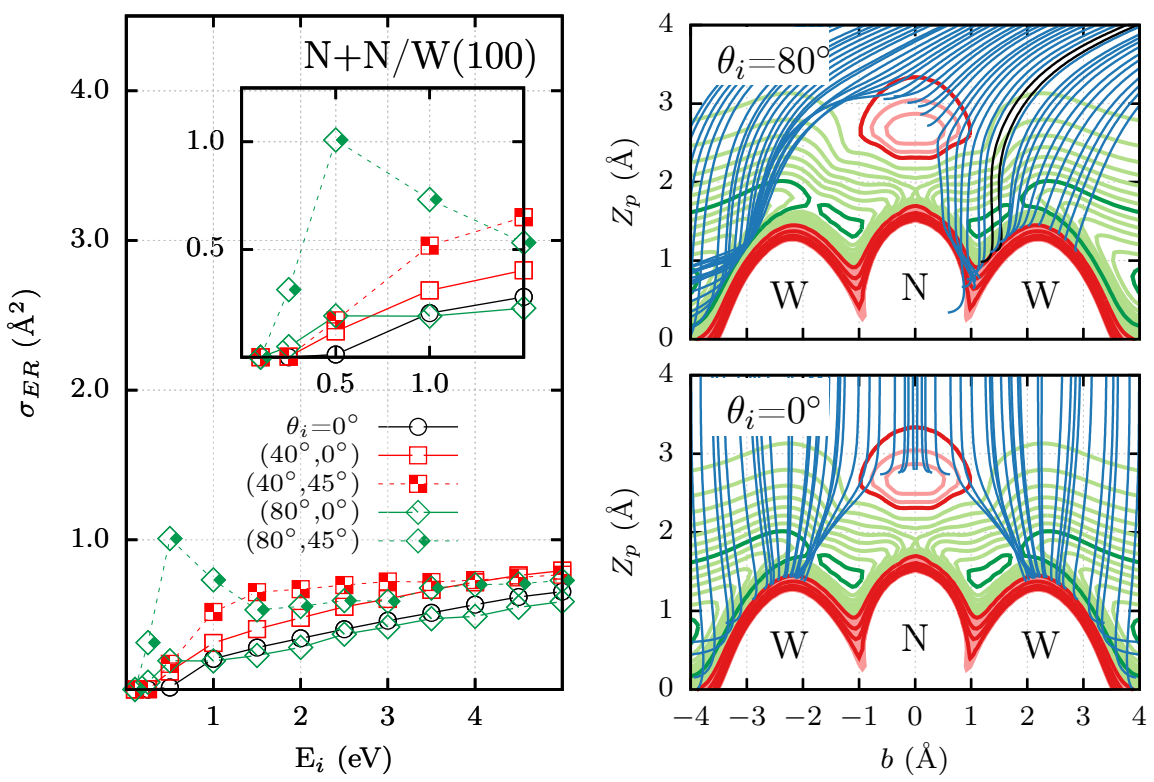

Figure 2: Left: ER abstraction cross section $\sigma_{E R}$ as a function of the projectile collision energy $\mathrm{E}_{i}$ for $\mathrm{N}+\mathrm{N} / \mathrm{W}(100)$. Different symbols correspond to different incidence angles $\left(\theta_{i}, \phi_{i}\right)$. Right: Projections of relevant projectile trajectories on the normal plane along the diagonal of the surface unit cell are plotted down to the first rebound together with the 2D contour plot of the PES in the same plane. The incidence conditions are $\mathrm{E}_{i}=0.5 \mathrm{eV}$, with $\theta_{i}=0^{\circ}$ (down) and with $\left(\theta_{i}=80^{\circ}, \phi_{i}=45^{\circ}\right)$ (up). Black (blue) trajectories are (non) reactive. Green (red) contour levels show negative (positive) values of the PES separated by $0.5 \mathrm{eV}$ $(0.2 \mathrm{eV})$. The zero potential energy (dark green contour level) is defined for the target at the bottom of the adsorption well and the projectile at $8 \AA$ from the surface.

mal incidence threshold is drastically reduced from $0.53 \mathrm{eV}$ at $\theta_{i}=0^{\circ}$ to $\sim 0.1 \mathrm{eV}$ at $\theta_{i}=80^{\circ}$. The analysis of the trajectories done in refs 36,38 for normal incidence and $\mathrm{E}_{i}<0.53 \mathrm{eV}$ showed that recombination at low $\mathrm{E}_{i}$ only stems from projectiles that abstract the target after bouncing on a neighboring tungsten atom. Such a mechanism involves mainly projectiles impinging the surface about the diagonal plane, i.e., the plane normal to the surface whose azimuth is $45^{\circ}$ (see dashed line in the surface unit cell of Figure 1). Here we find that the recombination dynamics for off-normal incidence and low collision energies proceeds in a similar way. Thus, the still prominent role of the diagonal plane also explains that the ER cross section is in general larger for $\phi_{i}=45^{\circ}$ than for $\phi_{i}=0^{\circ}$ for each of the off-normal $\theta_{i}$ studied here. Projections of the trajectories onto the diagonal plane reveal the dynamical origin of the significant increase of reactivity at $\left(\theta_{i}=80^{\circ}, \phi_{i}=45^{\circ}\right)$ and low collision energies 
$\left(\mathrm{E}_{i} \leq 0.5 \mathrm{eV}\right)$. Taking $\mathrm{E}_{i}=0.5 \mathrm{eV}$ as a representative example, the right panels of Figure 2 show these projections for the incoming part of the trajectories plotted on top of the 2D cut of the PES on the same plane. At normal incidence (Figure 2, down right), deflection of the impinging projectiles by the repulsive potential energy bump above the $\mathrm{N}$ adsorbate associated with the strong $\mathrm{N}-\mathrm{W}$ attraction, steers the projectile towards the neighboring empty cells, thus preventing the impinging atoms to enter in the occupied cell and recombine with the N-adsorbate. Conversely, upon increasing the polar angle to $\theta_{i}=80^{\circ}$, the topology of the interactions in the entrance channel clearly redirects the impinging projectiles towards the $\mathrm{W}$ atoms in the occupied unit cell and both $\mathrm{N}$ atoms may recombine (black lines on Figure 2, up right). As a result there is a significant increase in the ER reactivity.

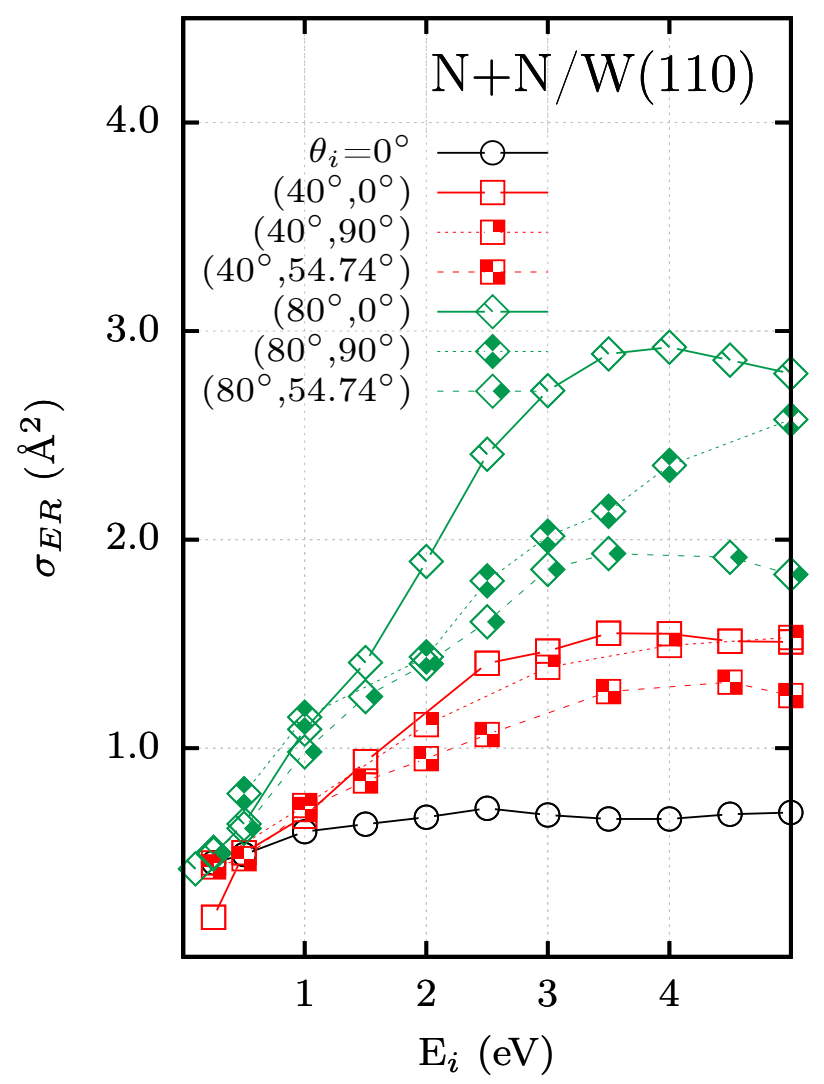

Figure 3: ER abstraction cross section $\sigma_{E R}$ as a function of the projectile collision energy $\mathrm{E}_{i}$ for $\mathrm{N}+\mathrm{N} / \mathrm{W}(110)$. Different symbols correspond to different $\left(\theta_{i}, \phi_{i}\right)$.

For $\mathrm{N}_{2}$ recombination on the $\mathrm{W}(110)$ surface, Figure 3 shows that the ER cross section 
significantly increases with $\theta_{i}$ above $\mathrm{E}_{i}=1.0 \mathrm{eV}$ and that such an increase depends on $\phi_{i}$. For instance, at $\mathrm{E}_{i}=5.0 \mathrm{eV}$, the cross section increases from $\sim 0.7 \AA^{2}$ at $\theta_{i}=0^{\circ}$ to $\sim 1.4 \AA^{2}$ at $\left(\theta_{i}=40^{\circ}, \phi_{i}=0^{\circ}\right)$ and $\sim 2.8 \AA^{2}$ at $\left(\theta_{i}=80^{\circ}, \phi_{i}=0^{\circ}\right)$. Figures 4 and 5 show a representative selection of the ER trajectories for $\mathrm{E}_{i}=3.0 \mathrm{eV}$ at normal and $\left(\theta_{i}=80^{\circ}, \phi_{i}=0^{\circ}\right)$ incidence, respectively, that are illustrative of the $\mathrm{ER}$ abstraction dynamics at $\mathrm{E}_{i}>3.0 \mathrm{eV}$. At normal incidence, ER reactions involving projectiles bouncing at impact parameters from the adsorbate position $b<3.17 \AA$ (close-ER, top panels) are discriminated from those involving higher impact parameters (far-ER, bottom panels). Both close-ER and far-ER with cross sections (probabilities) of $0.43 \AA^{2}(64 \%)$ and $0.25 \AA^{2}(36 \%)$, respectively, contribute significantly to the total ER abstraction process. We observe in Figure 4 by looking at the incoming part of the ER trajectories (green lines, $\mathrm{N}$-approach) that at this large incidence energy projectiles impinge the surface without being steered. Most of the impinging $\mathrm{N}$ atoms first collide with the $\mathrm{W}$ atoms in the topmost surface layer prior to abstracting the adsorbed $\mathrm{N}$ (target). Only about $10 \%$ of the projectiles react upon colliding on top of the target, i.e., with an impact parameter smaller than $0.75 \AA$ and an altitude higher than the target equilibrium altitude of $1.155 \AA$. The evolution of the trajectories during the first femtosecond after the rebound (short red lines in left panels) shows that the collision with the $\mathrm{W}$ atoms redirects the projectiles towards the target. Once the incoming and adsorbed $\mathrm{N}$ atoms recombine $(r<2.5$ $\AA$ ), the trajectories of the $\mathrm{CM}$ of the formed $\mathrm{N}_{2}$ (red lines in right panels) show that the nascent molecules leave the surface in directions roughly similar to the ones with which the projectiles are scattered from the $\mathrm{W}$ atoms.

At the most reactive $\left(\theta_{i}=80^{\circ}, \phi_{i}=0^{\circ}\right)$ incidence, we find that, in addition to the previously discussed close-ER and far-ER mechanisms, a new reaction pathway (grazing-ER) appears that no longer involves the collision of the projectile with a $\mathrm{W}$ atom prior recombining with the adsorbate. Figure 5 (bottom-left panels) shows that the projectile is first captured by the target and then collides just on top of the next $\mathrm{W}$ atom following the forward direction. The position of the target at the instant of the projectile rebound shows that the target has 

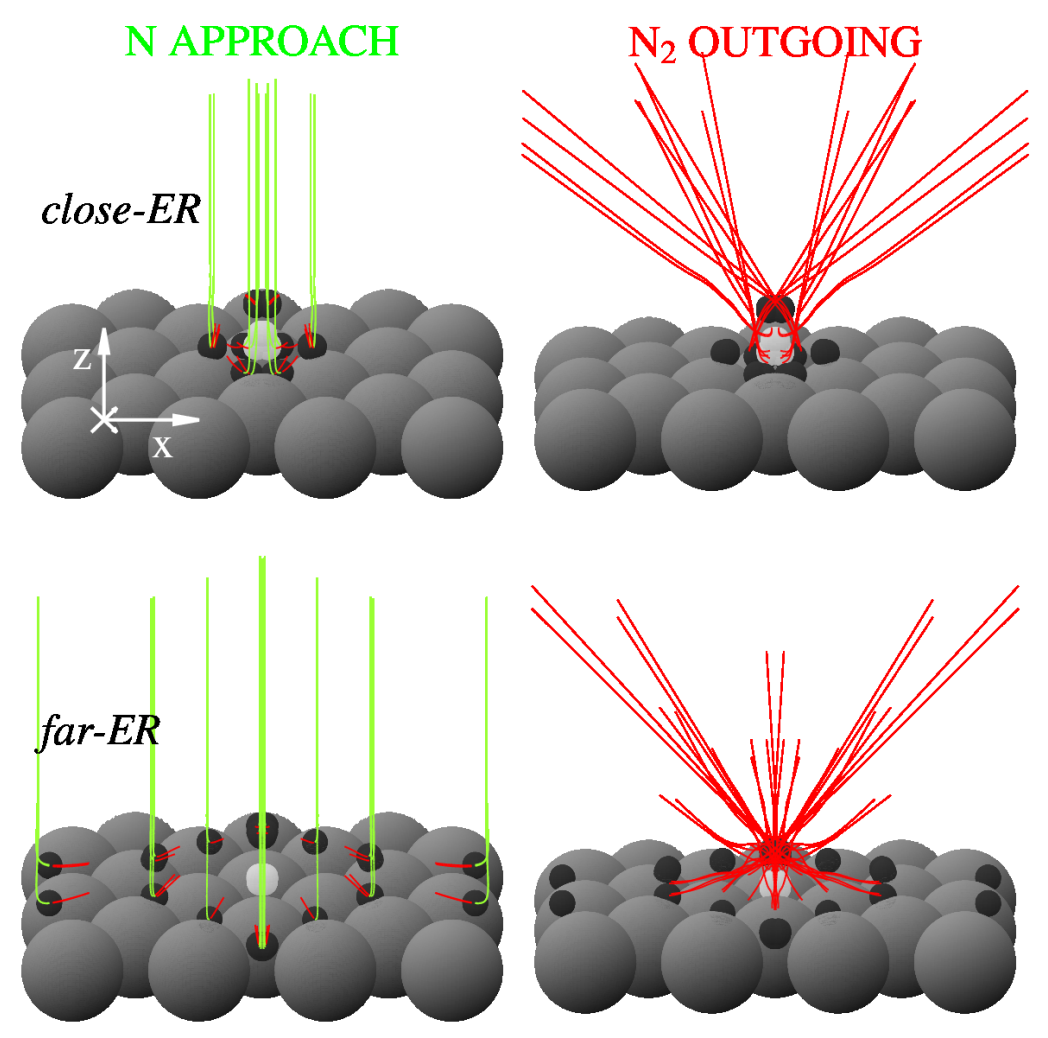

Figure 4: Close-ER (top panels) and far-ER (bottom panels) recombination of $\mathrm{N}_{2}$ on $\mathrm{W}(110)$ for an initial collision energy of $3.0 \mathrm{eV}$ and $\theta_{i}=0^{\circ}$. Left panels: trajectories of the projectiles down to the first rebound (green lines) and during the following one fs (red lines). Right panels: Diatom CM outgoing trajectories. Gray spheres represent surface W atoms. Black (white) spheres represent the projectile (target) position at the instant in which the projectile rebounds.

already been abstracted out of its adsorption site. This grazing-ER path is responsible of approx. $50 \%$ of the total ER cross section at these incidence conditions. The contribution of grazing-ER to the total reactivity increases with $\mathrm{E}_{i}$ until $\mathrm{E}_{i} \approx 3.0 \mathrm{eV}$. For higher incidence energies, the contribution of the three channels (grazing-ER with $\sim 50 \%$, close-ER with $35 \%$, and far-ER with $\sim 15 \%$ ) remains constant. The grazing-ER path is also observed at $\left(\theta_{i}=80^{\circ}, \phi_{i}=90^{\circ}\right)$ incidence, but its contribution to the total ER cross section is smaller. As in the previous case, the contribution of grazing-ER to the total reactivity increases with $\mathrm{E}_{i}$ until $\mathrm{E}_{i} \approx 3.5 \mathrm{eV}$ and then, the contribution of the three channels, namely, grazing-ER with $\sim 30 \%$, close-ER with $\sim 60 \%$, and far-ER with $\sim 10 \%$, remains constant. In contrast, 
close-ER
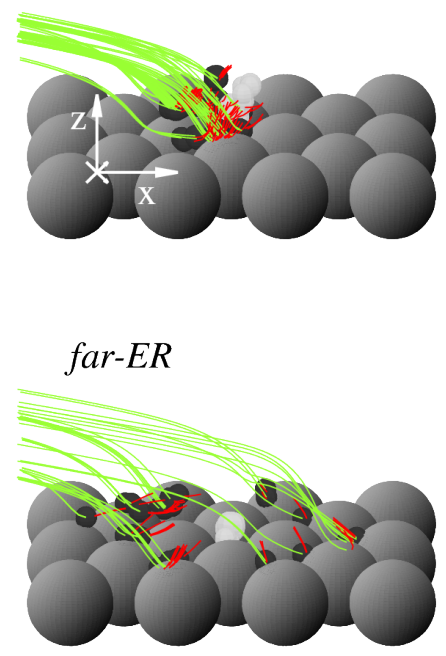

grazing-ER

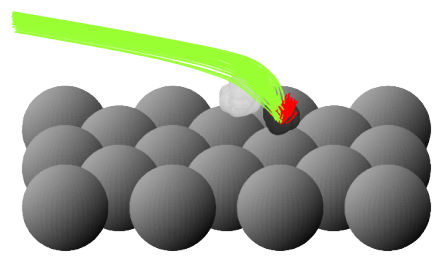

$\mathrm{N}_{2}$ OUTGOING
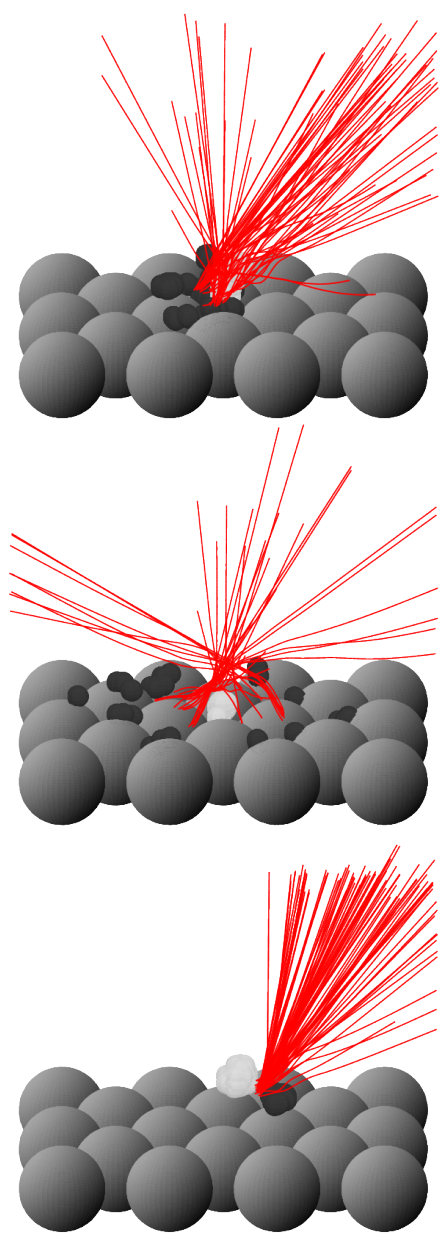

Figure 5: Same as Figure 4 for $\theta_{i}=80^{\circ}$ and $\phi_{i}=0^{\circ}$.

at $\left(\theta_{i}=80^{\circ}, \phi_{i}=54.74^{\circ}\right)$ incidence, the ER reaction proceeds similarly to normal incidence, i.e., only the close-ER and far-ER mechanisms are observed. At $\phi_{i}=54.74^{\circ}$ incidence there is not a close $\mathrm{W}$ atom in the forward direction of the projectiles that pass over the target and, therefore, grazing-ER is not observed for this incidence condition. At the intermediate $\theta_{i}=40^{\circ}$ the contribution of the grazing-ER mechanism decreases. At $\phi_{i}=0^{\circ}$ it is responsible of $\sim 15 \%$ of the total ER cross section at high $\mathrm{E}_{i}$, while at $\phi_{i}=90^{\circ}$ it contributes in $\sim 5 \%$ to the total reactivity. As observed for $\theta_{i}=80^{\circ}$, there are no grazing- $E R$ trajectories at $\phi_{i}=54.74^{\circ}$. Interestingly, a similar grazing- $E R$ mechanism was identified in the efficient $\mathrm{N}_{2}$ 
recombination on N-covered $\operatorname{Ag}(111) .{ }^{35,98}$

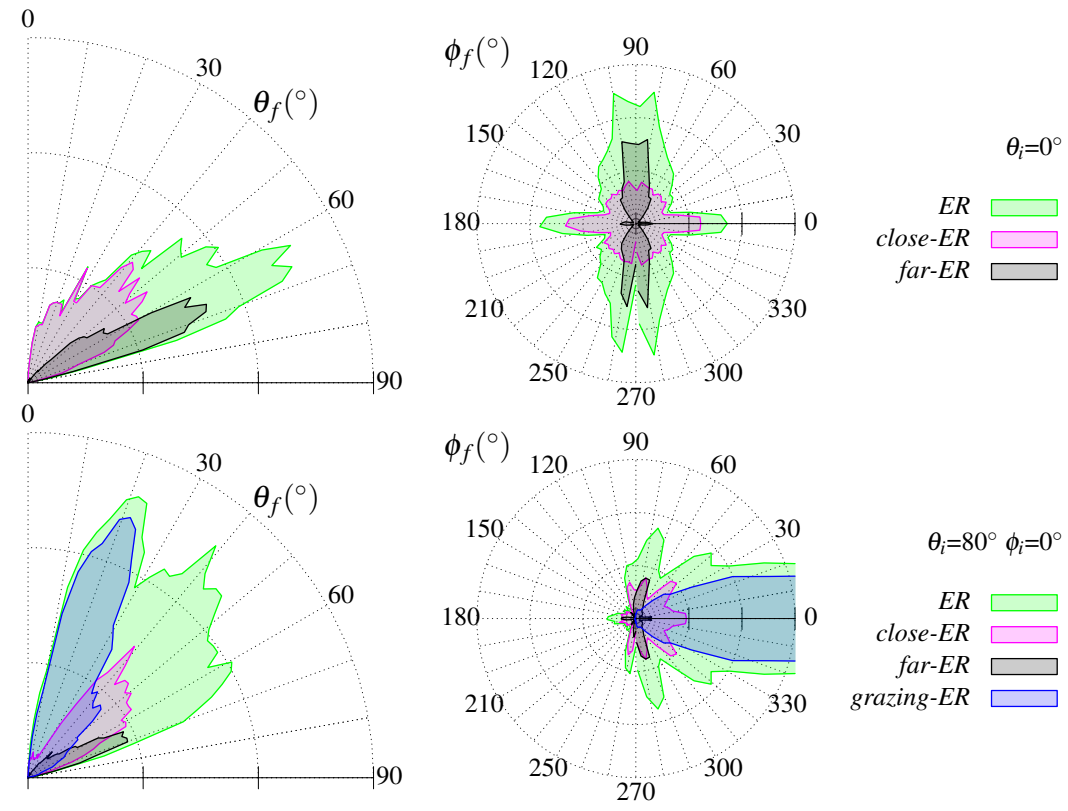

Figure 6: Final $\theta_{f}$ (left panels) and $\phi_{f}$ (right panels) angle distributions of the ER-formed $\mathrm{N}_{2}$ on $\mathrm{W}(110)$ for $\mathrm{E}_{i}=3.0 \mathrm{eV}$ and incidence angles: $\theta_{i}=0^{\circ}$ (top panels) and $\theta_{i}=80^{\circ}$ and $\phi_{i}=0^{\circ}$ (bottom panels). The total distribution (green) is plotted together with the contributions from close-ER (pink), far-ER (black), and grazing-ER (blue).

The angular distribution of the recombined products is one of the magnitudes that has been studied in the context of stereodynamical effects, as mentioned in the introduction. Figure 6 displays the polar (left) and azimuthal (right) angle distributions of the ER-formed $\mathrm{N}_{2}$ on $\mathrm{W}(110)$ for $\mathrm{E}_{i}=3.0 \mathrm{eV}$ and two different incidence angles, $\theta_{i}=0^{\circ}$ and $\left(\theta_{i}=80^{\circ}, \phi_{i}=0^{\circ}\right)$. Since the ER reaction occurs before the incoming atom equilibrates with the surface, the product is expected to retain "memory" of the parallel momentum of the projectile. Assuming a simple scattering picture, for off normal incidence one expects to find a forward peaked final azimuthal distribution. Although this is in general what it has been observed in most previous studies ${ }^{45,46,51,72,99,100}$ and here also for the ER-formed $\mathrm{N}_{2}$ molecules at $\left(\theta_{i}=80^{\circ}\right.$, $\phi_{i}=0^{\circ}$ ) incidence, we have also observed just the opposite. For instance, the ER-formed $\mathrm{N}_{2}$ molecules on $\mathrm{W}(100)$ at $\mathrm{E}_{i}=1.0 \mathrm{eV}$ and $\left(\theta_{i}=80^{\circ}, \phi_{i}=45^{\circ}\right)$ have a backward peaked angular distribution (not shown). Focusing now on the polar angle distributions for the $\mathrm{N}_{2}$ recombi- 
nation on W(110) (left panels of Figure 6), the larger $\theta_{f}$ values obtained at normal incidence show that a larger initial parallel momentum does not necessarily imply a larger final parallel momentum in the formed molecules. By analyzing the polar angle distribution for each of the three ER mechanisms characterized above, we observe that the dependence of $\theta_{f}$ on $\theta_{i}$ mainly comes from the new grazing-ER pathway. Clearly, the close-ER and far-ER polar distributions are not significantly dependent on the incidence conditions. In contrast, the grazing-ER mechanism appearing at large off-normal incidence angles involve much narrower $\theta_{f^{-}}$and $\phi_{f}$-distributions that are centered at $20^{\circ}$ and $0^{\circ} \pm 15^{\circ}$, i.e., along the forward direction. In conclusion, our results show that although the ER-formed molecules retain "memory" of the incidence angles, the complexity of the PES is such that the dynamics cannot be described with a simple scattering picture. To our knowledge, experimental works analyzing the angle distributions of the abstracted molecules at off-normal incidence conditions such as $\mathrm{HD}$ from $\mathrm{Cu}(111),{ }^{45,51,99} \mathrm{HCl}$ from $\mathrm{Au}(111),{ }^{46,100}$ and $\mathrm{NO}$ from $\mathrm{Ru}(1000){ }^{72}$ found forward peaked azimuthal distributions. The additional analysis of $\theta_{f}$ for various $\theta_{i}$ performed in ref 72 showed that there was no a clear correlation between the final and initial incidence angles. As done here, this finding was interpreted as a clear indication of the complex topology of the gas-adsorbate interaction.

In comparison with $\mathrm{N}$ abstraction, Figure 7 shows that the dependence on the incidence angle of the $\mathrm{H}_{2}$ ER cross section on both tungsten surfaces is quantitatively smaller. Nevertheless, a slight sensitivity to the incidence angle is observed for $\mathrm{H}+\mathrm{H} / \mathrm{W}(100)$, in particular at $\theta_{i}=80^{\circ}$. Such a specific behavior depends on an intricate competition between two ER abstraction mechanisms involving rebounds of the projectile on $\mathrm{W}$ atoms from the first and second topmost layers. ${ }^{79}$ Apart from it, there are no remarkable new features in the recombination paths that can be attributed to the off-normal incidence angles as it was the case for $\mathrm{N}_{2}$ recombination. Regarding the polar and azimuthal angle distributions of the formed $\mathrm{H}_{2}$ we also obtain that there is not a clear correlation between the incidence and outgoing angles. Independently of the azimuthal incidence angle, the $\theta_{f}$-distributions are 


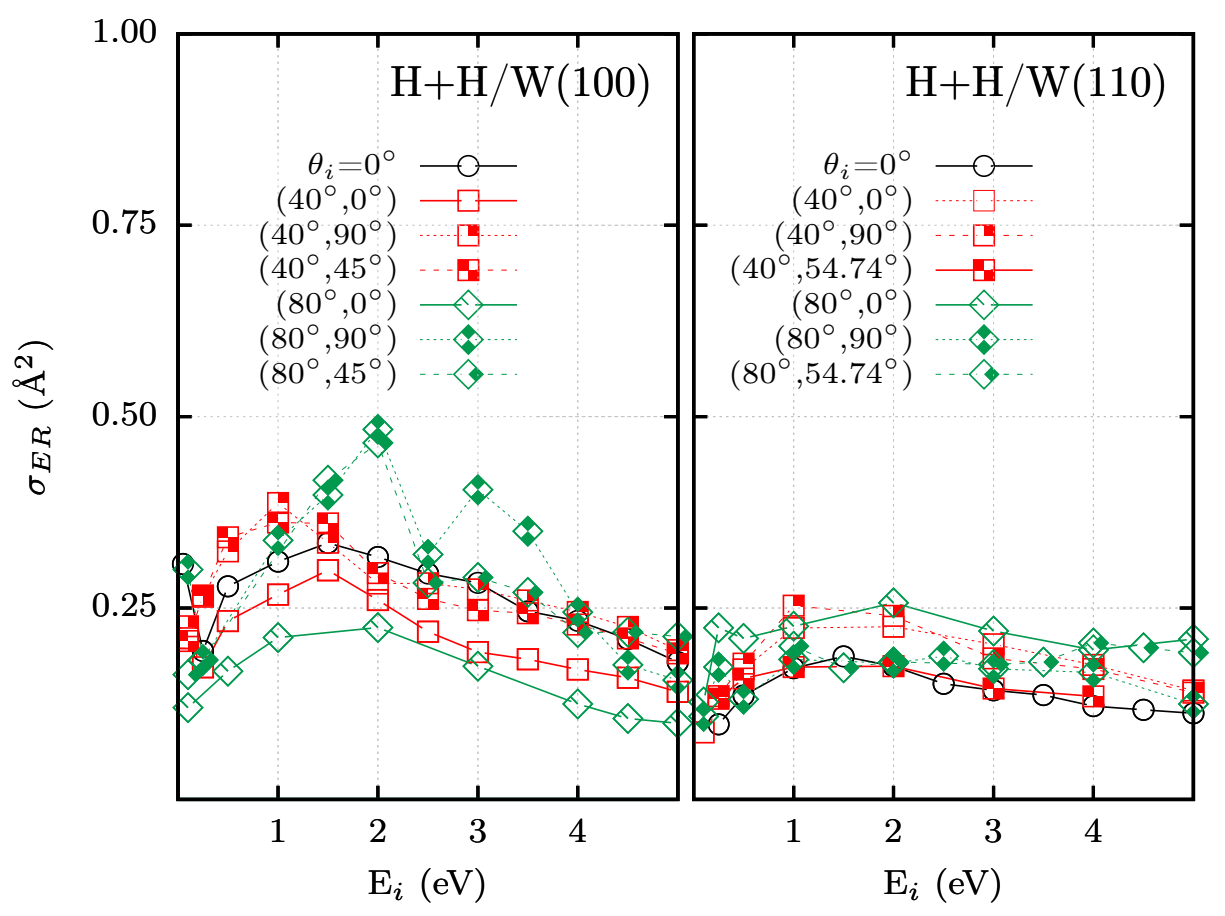

Figure 7: ER abstraction cross section $\sigma_{E R}$ as a function of the projectile's collision energy $\mathrm{E}_{i}$ for $\mathrm{H}+\mathrm{H} / \mathrm{W}(100)$ and $\mathrm{H}+\mathrm{H} / \mathrm{W}(110)$. The $\left(\theta_{i}, \phi_{i}\right)$ incidence angles are indicated in each plot.

centered around $45^{\circ}-50^{\circ}\left(40^{\circ}\right)$ on the $\mathrm{W}(100)$ ( W (110)) surface. The azimuthal distributions show preference for certain $\phi_{f}$ values, but in general the forward direction is not particularly favored.

\subsection{LDFA-GLO results: Energy loss effects at off-normal inci- dence}

In order to quantify the energy dissipated to the metal as well as its dependence on the incidence angle, calculations have also been carried out within the LDFA-GLO scheme, using a surface temperature of $300 \mathrm{~K}$ in all the studied systems. The BOSS and LDFAGLO ER cross sections as a function of $\mathrm{E}_{i}$ are compared in Figures 8 and 9 for $\mathrm{H}_{2}$ and $\mathrm{N}_{2}$ recombination, respectively. As obtained for normal incidence, ${ }^{66}$ the BOSS and the LDFAGLO cross sections are qualitatively the same for each recombination process and incidence 
angle. Sizable quantitative differences between the adiabatic and nonadiabatic pictures only appear for $\mathrm{N}_{2}$ recombination, in particular for $\mathrm{N}+\mathrm{N} / \mathrm{W}(110)$, for which reactivity sensibly decreases when including energy dissipation, especially at large off-normal angles.

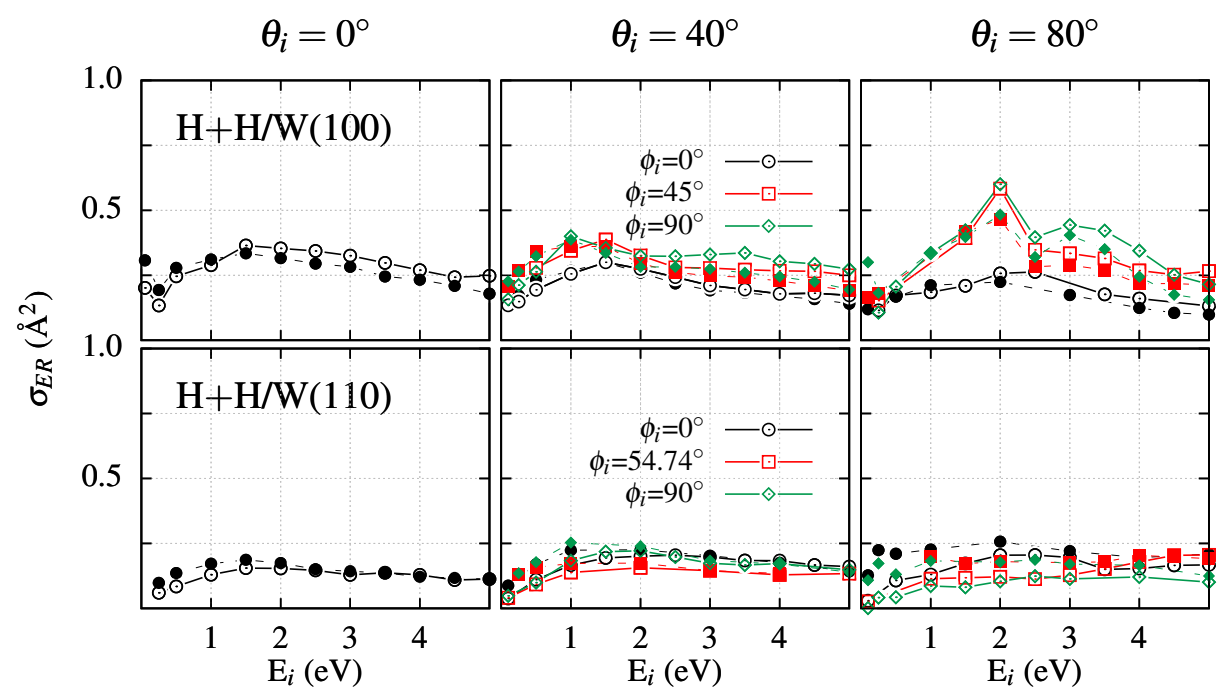

Figure 8: ER recombination cross section $\sigma_{E R}$ for $\mathrm{H}+\mathrm{H} / \mathrm{W}(100)$ (top panels) and $\mathrm{H}+\mathrm{H} / \mathrm{W}(110)$ (bottom panels) as a function of the projectile's collision energy $\mathrm{E}_{i}$ as obtained from BOSS (solid symbols) and LDFA-GLO (empty symbols) simulations. Different symbols correspond to incidence angles $\left(\theta_{i}, \phi_{i}\right)$.

We have also examined possible incidence angle effects on the total energy dissipated into the surface as well as on the contribution of each energy dissipation channel. The contribution from $e-h$ pair excitations $\Delta \mathrm{E}_{e h}$ is computed for each trajectory as

$$
\Delta \mathrm{E}_{e h}=\sum_{i, n} \eta\left(\mathbf{r}_{i}\right)\left|\frac{d \mathbf{r}_{i}}{d t}\right|^{2} \Delta t_{n}
$$

where the subscript $i$ refers to the projectile and target atoms, $\eta\left(\mathbf{r}_{i}\right)$ are the corresponding friction coefficients, and $\Delta t_{n}$ is the time interval at the $n^{t h}$ integration step. After averaging $\Delta \mathrm{E}_{e h}$ over all ER trajectories, the average energy lost into phonons $\left\langle\Delta \mathrm{E}_{p h}\right\rangle$ is computed by subtracting $\left\langle\Delta \mathrm{E}_{e h}\right\rangle$ to the total LDFA-GLO average energy loss $\langle\Delta \mathrm{E}\rangle$. The results for $\mathrm{H}$ and $\mathrm{N}$ abstraction are shown in Figures 10 and 11, respectively.

For $\mathrm{H}$ abstraction and regardless of the incidence angle and crystal face, the energy loss 


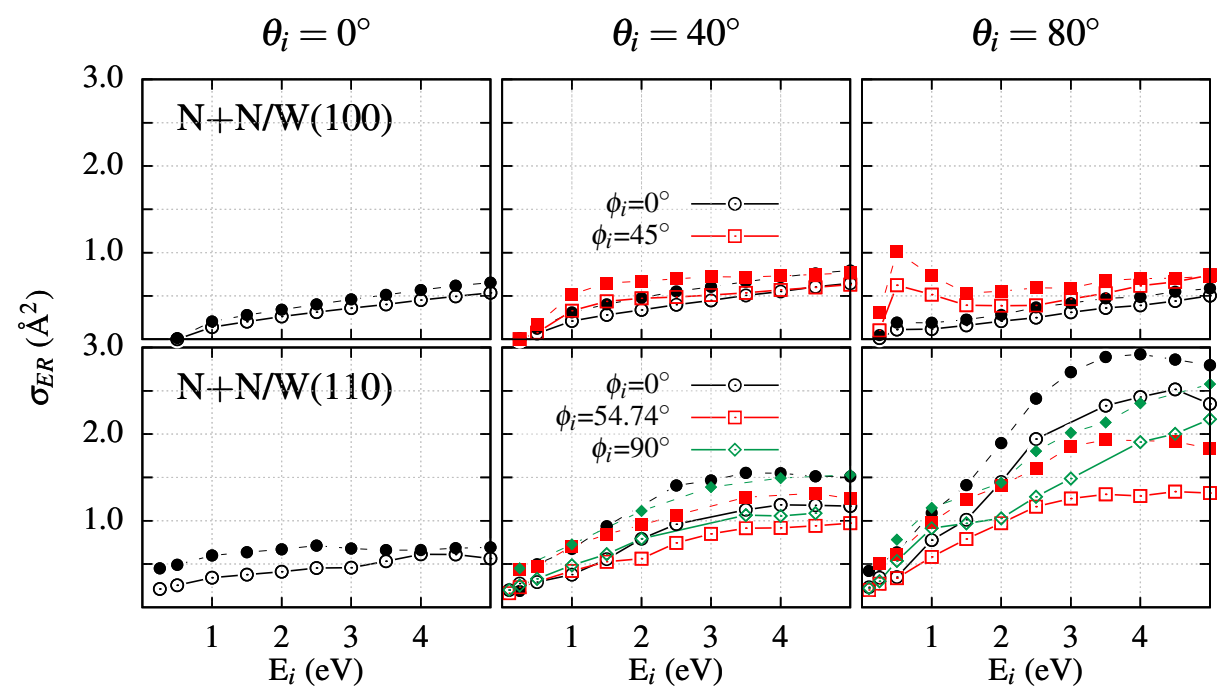

Figure 9: Same as Figure 8 but for N+N/W(100) (top panels) and N+N/W(110) (bottom panels).

is roughly $0.5 \mathrm{eV}$ at the lowest $\mathrm{E}_{i}=0.1 \mathrm{eV}$ and increases to around $1.0 \mathrm{eV}$ at $\mathrm{E}_{i}=5.0 \mathrm{eV}$. In all cases, more than $90 \%$ of the total energy loss is due to electronic excitations, whereas the energy dissipated into phonon excitations is marginal and represents less than $10 \%$ of the total energy loss. Figure 11 shows that in the case of $\mathrm{N}_{2}$ recombination, $\langle\Delta \mathrm{E}\rangle$ varies from 0.5 to $2 \mathrm{eV}$, i.e., compared to the previous $\mathrm{H}_{2}$ case, the energy losses are about a factor 1.5-2 larger at the largest $E_{i}$. On both tungsten surfaces, the energy losses are predominantly due to phonon excitations that accounts for $71-82 \%$ of the total energy loss. Note that this energy dissipation channel is responsible of the decrease of $\langle\Delta \mathrm{E}\rangle$ as $\theta_{i}$ increase. Such a dependence is partially related to the appearance of the grazing-ER mechanism at large $\theta_{i}$, for which the energy exchange with the surface lattice seems to be smaller than for the far-ER and close-ER recombination processes.

Finally, the influence of the incidence angle on the distribution of energy into the internal motion of the formed molecules has been investigated within the LDFA-GLO scheme. The dependence of the average internal energies on the incidence angle for ER-formed $\mathrm{H}_{2}$ molecules is negligible. Moreover, the internal energy distributions of the $\mathrm{H}_{2}$ molecules are insensitive to the crystal face. The same conclusion was obtained in ref 66 for normal in- 


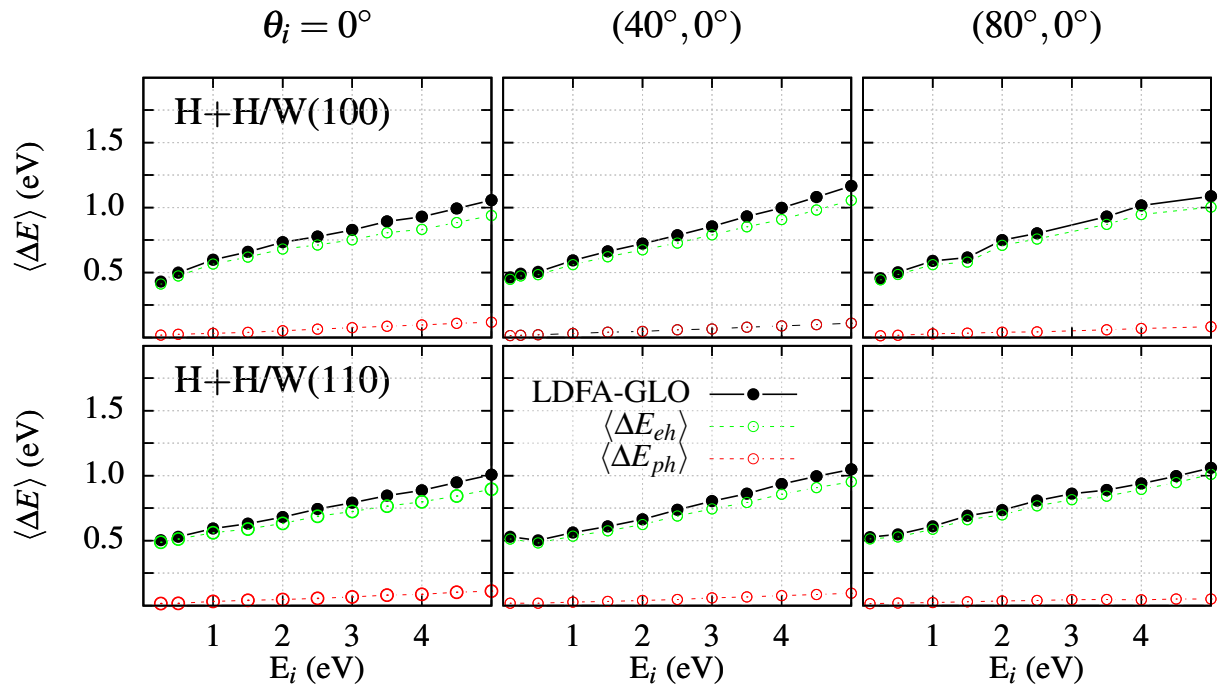

Figure 10: Average energy loss $\langle\Delta \mathrm{E}\rangle$ as a function of the projectile's collision energy $\mathrm{E}_{i}$ obtained from the LDFA-GLO (black) simulations. The average energy loss into phonons $\left\langle\Delta \mathrm{E}_{p h}\right\rangle$ (red) and into $e-h$ pair excitations $\left\langle\Delta \mathrm{E}_{e h}\right\rangle$ (green) are also shown.

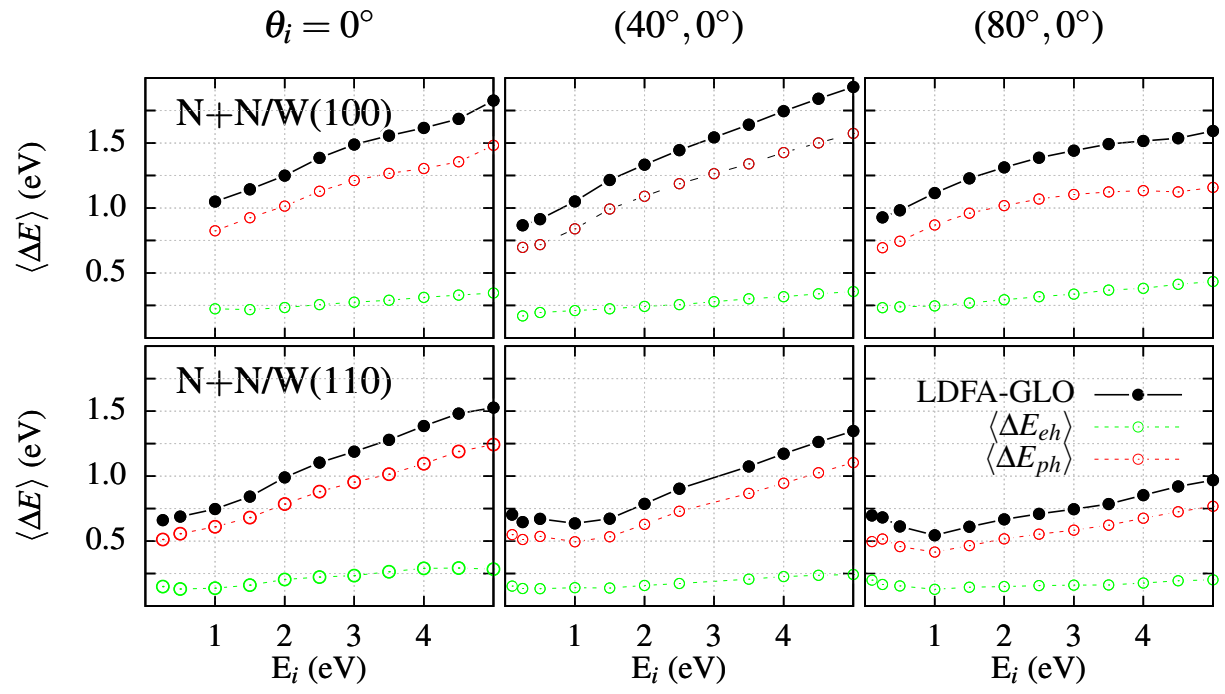

Figure 11: Same as Figure 10 but for $\mathrm{N}+\mathrm{N} / \mathrm{W}(100)$ and $\mathrm{N}+\mathrm{N} / \mathrm{W}(110)$. 
cidence. On the contrary, a more pronounce dependence is obtained in the ER-formed $\mathrm{N}_{2}$, particularly on the W(110) surface. Figure 12 shows that on this surface the average final translational, vibrational, and rotational energies depend on the incidence angle for all the $\mathrm{E}_{i}$ range. Differences as large as $0.7 \mathrm{eV}$ in the internal energy distribution can be observed at given incidence angles $\left(\theta_{i}, \phi_{i}\right)$ and $\mathrm{E}_{i}$.

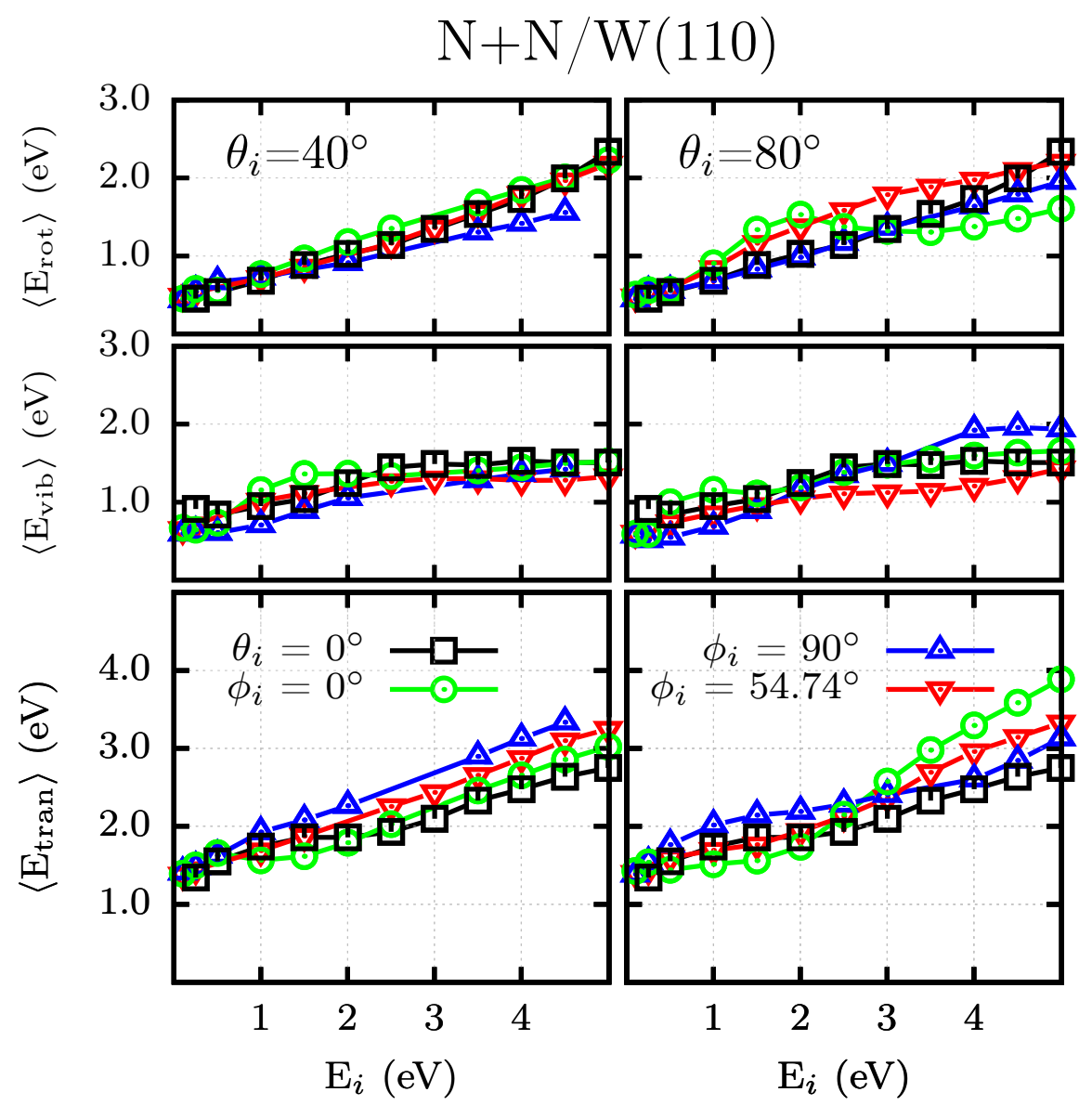

Figure 12: Average final translational $\left\langle\mathrm{E}_{\text {tran }}\right\rangle$, vibrational $\left\langle\mathrm{E}_{\mathrm{vib}}\right\rangle$, and rotational energies $\left\langle\mathrm{E}_{\text {rot }}\right\rangle$ of the ER-formed $\mathrm{N}_{2}$ molecules on W(110) as a function of the initial collision energy $\mathrm{E}_{i}$ for $\theta_{i}=40^{\circ}$ (left panels) and $\theta_{i}=80^{\circ}$ (right panels). Different symbols correspond to different $\phi_{i}$. For comparison, the results at normal incidence (empty squares) are included in all panels.

The main differences in the final energy distributions of the ER-formed molecules are due to the dynamical changes caused by the incidence angle that were discussed above. For instance, for $\mathrm{N}_{2}$ recombination on $\mathrm{W}(110)$, where we identified the largest change in the recombination paths with the appearance of the grazing-ER mechanism, the rotational 

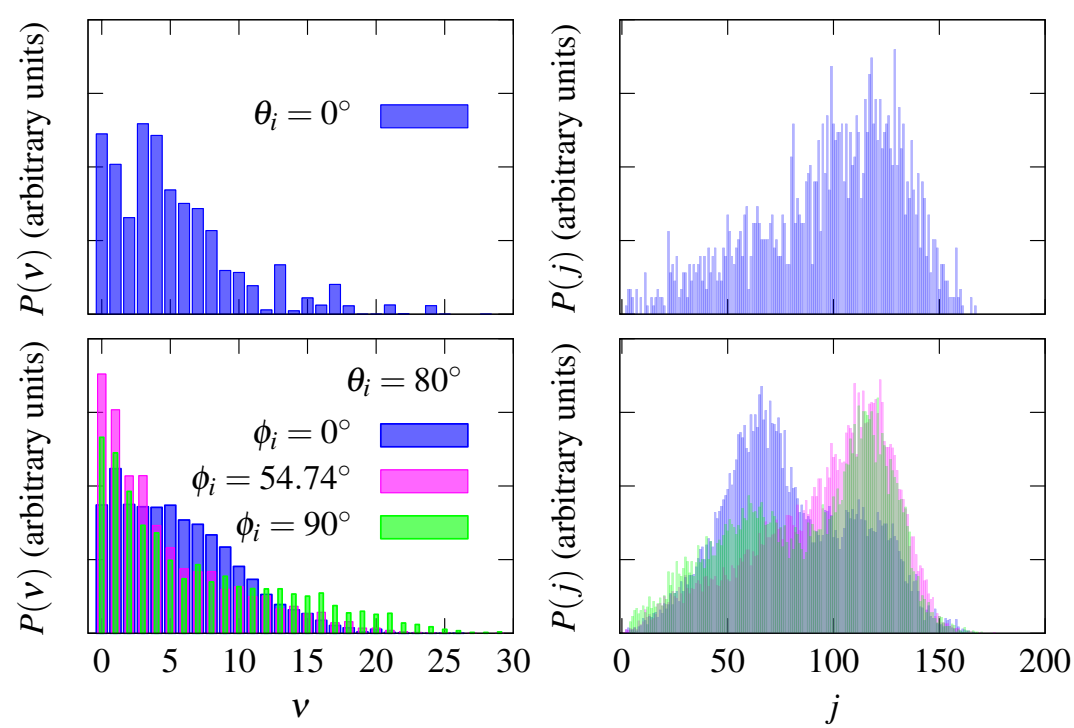

Figure 13: Normalized vibrational (left) and rotational (right) states distributions of the ERformed $\mathrm{N}_{2}$ molecules on $\mathrm{W}(110)$ for $\mathrm{E}_{i}=5.0 \mathrm{eV}$ at $\theta_{i}=0^{\circ}$ (upper panels) and $\theta_{i}=80^{\circ}$ (bottom panels).

state distribution is characterized by different shapes depending on the incidence angle, as observed in Figure 13 for $\mathrm{E}_{i}=5.0 \mathrm{eV}$. The rotational distributions at normal and $\left(\theta_{i}=80^{\circ}\right.$, $\left.\phi_{i}=54.74^{\circ}\right)$ incidence are very similar. Both distributions cover the range 0-160 $j$ and exhibit a peak around $j=125$. However, for $\left(\theta_{i}=80^{\circ}, \phi_{i}=0^{\circ}\right)$ and $\left(\theta_{i}=80^{\circ}, \phi_{i}=90^{\circ}\right)$, an additional peak appears at lower $j$. We have checked that this low $j$ peak is originated mainly by grazing-ER molecules, what explains why this peak is also higher for $\left(\theta_{i}=80^{\circ}, \phi_{i}=0^{\circ}\right)$. Regarding the vibrational distributions, we observe that there is an important fraction of highly vibrationally excited molecules at $\left(\theta_{i}=80^{\circ}, \phi_{i}=0^{\circ}\right)$ and $\left(\theta_{i}=80^{\circ}, \phi_{i}=90^{\circ}\right)$ that are also formed by the grazing-ER mechanism.

\section{Conclusions}

We have studied the Eley-Rideal recombination of $\mathrm{H}_{2}$ and $\mathrm{N}_{2}$ on W(100) and $\mathrm{W}(110)$ for different incidence angles and energies of the incoming atom. The analysis of the ER cross sections, energy dissipation into the metal, and energy distribution of the formed molecules show a general stronger dependence on the incidence angle for $\mathrm{N}_{2}$ than for $\mathrm{H}_{2}$ recombination. 
Non negligible quantitative changes on the reactivity are found in the four systems, at least for the highest incidence polar angle. Nevertheless, high qualitative changes on ER reactivity are only found for $\mathrm{N}_{2}$ recombination. On the one hand, the energy threshold for $\mathrm{N}$ abstraction from $\mathrm{W}(100)$ is drastically reduced with the polar incidence angle. On the other hand, very efficient $\mathrm{N}$ ER abstraction is observed on $\mathrm{W}(110)$ at the highest $\theta_{i}$ due to the appearance of a new recombination pathway, grazing-ER, which has been characterized in detail. These incidence angle effects have a purely dynamical origin, which is related to the high corrugation of the PES on both systems.

The energy dissipated to the metal via phonon excitations and low energy $e-h$ pair excitations is analyzed as well as the effect of these two processes in the reactivity. The main conclusions of the same analysis at normal incidence remain. ${ }^{66}$ The average energy losses upon ER recombination of $\mathrm{H}$ and $\mathrm{N}$ on the $\mathrm{H}-$ and $\mathrm{N}-\mathrm{W}(100)$ and $\mathrm{W}(110)$ surfaces are within the range of 0.5-2.0 eV. In general, the energy loss increases with $\mathrm{E}_{i}$ but its dependence on the incidence angle of the projectile is minor.

The partition of energy into the degrees of freedom of the ER-formed diatom does not depend on incidence angle for $\mathrm{H}$ abstraction on tungsten. For $\mathrm{N}_{2}$ recombination, stereodynamical effects are small for W(100) but significant for W(110) at high collision energy. The reason is that such effects essentially originate from the grazing-ER mechanism that only works on the W(110) surface.

\section{Acknowledgement}

O.G., J.I.J, and M.A. acknowledge financial support by the Basque Departamento de Educación, Universidades e Investigación, the University of the Basque Country UPV/EHU (Grant No IT-756-13) and the Spanish Ministerio de Economía y Competitividad (Grant No. FIS2013-48286-C2-2-P and FIS2016-76471-P). O.G., M.A., and P.L. acknowledge the IDEX Bordeaux (ANR-10-IDEX-03-02) and Euskampus for fundings. Computational resources were provided by the DIPC computing center, the Mésocentre de Calcul Intensif 
Aquitain (MCIA). Research is conducted in the scope of the Transnational Common Laboratory "QuantumChemPhys: Theoretical Chemistry and Physics at the Quantum Scale".

\section{References}

(1) Szekely, J. Gas-Solid Reactions; Elsevier Science, 2012.

(2) Pearson, C. Conservation of Marine Archaeological Objects; Elsevier Science, 2014.

(3) Sakintuna, B.; Lamari-Darkrim, F.; Hirscher, M. Metal Hydride Materials for Solid Hydrogen Storage: A Review. Int. J. Hydrogen Energ. 2007, 32, 1121 - 1140.

(4) Honkala, K.; Hellman, A.; Remediakis, I.; Logadottir, A.; Carlsson, A.; Dahl, S.; Christensen, C.; Nørskov, J. Ammonia Synthesis from First-Principles Calculations. Science 2005, 307, 555-558.

(5) Rayment, T.; Schlögl, R.; Thomas, J. M.; Ertl, G. Structure of the Ammonia Synthesis Catalyst. Nature 1985, 315, 311-313.

(6) Somorjai, G. A. Introduction to Surface Chemistry and Catalysis; Wiley, New York, 1994.

(7) Federici, G.; Wuerz, H.; Janeschitz, G.; Tivey, R. Erosion of Plasma-Facing Components in ITER. Fusion Eng. Des. 2002, 61-62, 81-94.

(8) Federici, G.; Andrew, P.; Barabaschi, P.; Brooks, J.; Doerner, R.; Geier, A.; Herrmann, A.; Janeschitz, G.; Krieger, K.; Kukushkin, A. et al. Key ITER Plasma Edge and Plasma-Material Interaction Issues. J. Nucl. Mater. 2003, 313, 11-22.

(9) Greenberg, J. M. Cosmic Dust and our Origins. Surf. Sci. 2002, 500, 793-822.

(10) Molina, M. J.; Molina, L. T.; Golden, D. M. Environmental Chemistry (Gas and Gas-Solid Interactions): The Role of Physical Chemistry. J. Phys. Chem. 1996, 100, $12888-12896$. 
(11) Mathis, J. S. Observations and Theories of Interstellar Dust. Rep. Prog. Phys. 1993, $56,605-652$.

(12) Winnewisser, G.; Herbst, E. Interstellar Molecules. Rep. Prog. Phys. 1993, 56, 12091273.

(13) Guvenc, Z. B.; Sha, X.; Jackson, B. Eley-Rideal and Hot Atom Reactions between Hydrogen Atoms on Ni(100): Electronic Structure and Quasiclassical Studies. J. Chem. Phys. 2001, 115, 9018-9027.

(14) Shalashilin, D. V.; Jackson, B.; Persson, M. Eley-Rideal and Hot-Atom Reactions of $\mathrm{H}(\mathrm{D})$ Atoms with $\mathrm{D}(\mathrm{H})$-covered $\mathrm{Cu}(111)$ Surfaces; Quasiclassical Studies. J. Chem. Phys. 1999, 110, 11038-11046.

(15) Caratzoulas, S.; Jackson, B.; Persson, M. Eley-Rideal and Hot-Atom Reaction Dynamics of $\mathrm{H}(\mathrm{g})$ with $\mathrm{H}$ Adsorbed on $\mathrm{Cu}(111)$. J. Chem. Phys. 1997, 107, 6420-6431.

(16) Guvenc, Z. B.; Sha, X.; Jackson, B. The Effects of Lattice Motion on Eley-Rideal and Hot Atom Reactions: Quasiclassical Studies of Hydrogen Recombination on $\mathrm{Ni}(100)$. J. Phys. Chem. B 2002, 106, 8342-8348.

(17) Jackson, B.; Persson, M. Vibrational Excitation in Recombinative Desorption of Hydrogen on Metal Surfaces: Eley-Rideal Mechanism. Surf. Sci. 1992, 269, 195-200.

(18) Jackson, B.; Lemoine, D. Eley-Rideal Reactions Between H Atoms on Metal and Graphite Surfaces: The Variation of Reactivity with Substrate. J. Chem. Phys. 2001, $114,474-482$.

(19) Jackson, B.; Sha, X.; Guvenc, Z. B. Kinetic Model for Eley-Rideal and Hot Atom Reactions between H Atoms on Metal Surfaces. J. Chem. Phys. 2002, 116, 25992608. 
(20) Kalyanaraman, C.; Lemoine, D.; Jackson, B. Eley-Rideal and Hot-Atom Reactions between Hydrogen Atoms on Metals: Quantum Mechanical Studies. Phys. Chem. Chem. Phys. 1999, 1, 1351-1358.

(21) Kratzer, P.; Brenig, W. Highly Excited Molecules from Eley-Rideal Reactions. Surf. Sci. 1991, 254, 275-280.

(22) Lanzani, G.; Martinazzo, R.; Materzanini, G.; Pino, I.; Tantardini, G. F. Chemistry at Surfaces: from Ab Initio Structures to Quantum Dynamics. Theor. Chem. Acc. 2007, $117,805-825$.

(23) Lemoine, D.; Quattrucci, J. G.; Jackson, B. Efficient Eley-Rideal Reactions of H Atoms with Single Cl Adsorbates on Au(111). Phys. Rev. Lett. 2002, 89, 268302.

(24) Martinazzo, R.; Assoni, S.; Marinoni, G.; Tantardini, G. F. Hot-atom versus EleyRideal Dynamics in Hydrogen Recombination on $\mathrm{Ni}(100)$. I. The Single-adsorbate Case. J. Chem. Phys. 2004, 120, 8761-8771.

(25) Persson, M.; Jackson, B. Flat Surface Study of the Eley-Rideal Dynamics of Recombinative Desorption of Hydrogen on a Metal Surface. J. Chem. Phys. 1995, 102, 1078-1093.

(26) Quattrucci, J. G.; Jackson, B.; Lemoine, D. Eley-Rideal Reactions of H atoms with Cl Adsorbed on $\mathrm{Au}$ (111): Quantum and Quasiclassical Studies. J. Chem. Phys. 2003, 118, 2357-2366.

(27) Rutigliano, M.; Cacciatore, M. Eley-Rideal Recombination of Hydrogen Atoms on a Tungsten Surface. Phys. Chem. Chem. Phys. 2011, 13, 7475-7484.

(28) Shalashilin, D. V.; Jackson, B. Formation and Dynamics of Hot-precursor Hydrogen Atoms on Metal Surfaces: Trajectory Simulations and Stochastic Models. J. Chem. Phys. 1998, 109, 2856-2864. 
(29) Shin, H. K. Vibrationally Excited Molecules from the Reaction of H Atoms and Chemisorbed H Atoms on a Metal Surface. Chem. Phys. Lett. 1995, 244, 235-244.

(30) Tully, J. C. Dynamics of Gas-Surface Interactions: Reaction of Atomic Oxygen with Adsorbed Carbon on Platinum. J. Chem. Phys. 1980, 73, 6333-6342.

(31) Stampfl, C.; Scheffler, M. Density-Functional Theory Study of the Catalytic Oxidation of CO over Transition Metal Surfaces. Surf. Sci. 1999, 433, 119-126.

(32) Kim, W. K.; Ree, J.; Shin, H. K. Eley-Rideal Dynamics of the Chlorine Atom Abstraction of Hydrogen Chemisorbed on Silicon. J. Phys. Chem. A 1999, 103, 411-419.

(33) Cacciatore, M.; Rutigliano, M.; Billing, G. D. Eley-Rideal and Langmuir-Hinshelwood Recombination Coefficients for Oxygen on Silica Surfaces. J. Thermophys. Heat Transfer 1999, 13, 195-203.

(34) Quattrucci, J. G.; Jackson, B. Quasiclassical Study of Eley-Rideal and Hot Atom Reactions of $\mathrm{H}$ Atoms with $\mathrm{Cl}$ Adsorbed on a $\mathrm{Au}(111)$ Surface. J. Chem. Phys. 2005, 122, 074705 .

(35) Blanco-Rey, M.; Díaz, E.; Bocan, G. A.; Díez Muiño, R.; Alducin, M.; Juaristi, J. I. Efficient $\mathrm{N}_{2}$ Formation on $\mathrm{Ag}(111)$ by Eley-Rideal Recombination of Hyperthermal Atoms. J. Phys. Chem. Lett. 2013, 4, 3704-3709.

(36) Quintas-Sánchez, E.; Larrégaray, P.; Crespos, C.; Martin-Gondre, L.; RubayoSoneira, J.; Rayez, J. C. Dynamical Reaction Pathways in Eley-Rideal Recombination of Nitrogen from W(100). J. Chem. Phys. 2012, 137, 064709.

(37) Quintas-Sánchez, E.; Crespos, C.; Larrégaray, P.; Rayez, J. C.; Martin-Gondre, L.; Rubayo-Soneira, J. Surface Temperature Effects on the Dynamics of $\mathrm{N}_{2}$ Eley-Rideal Recombination on W(100). J. Chem. Phys. 2013, 138, 024706. 
(38) Quintas-Sánchez, E.; Larrégaray, P.; Crespos, C. Influence of Surface Symmetry on the Onset of Nitrogen Eley-Rideal Recombination on Tungsten. J. Phys. Chem. C 2014, 118, 12224-12229.

(39) Persson, M.; Jackson, B. Isotope Effects in the Eley-Rideal Dynamics of the Recombinative Desorption of Hydrogen on a Metal Surface. Chem. Phys. Lett. 1995, 237, 468-473.

(40) Pétuya, R.; Larrégaray, P.; Crespos, C.; Aurel, P.; Busnengo, H. F.; Martínez, A. E. Scattering of Atomic Hydrogen Off a H-Covered W(110) Surface: Hot-Atom versus Eley-Rideal Abstraction Dynamics. J. Phys. Chem. C 2015, 6, 3171-3179.

(41) Pétuya, R.; Larrégaray, P.; Crespos, C.; Busnengo, H. F.; Martínez, A. E. Dynamics of $\mathrm{H}_{2}$ Eley-Rideal Abstraction from W(110): Sensitivity to the Representation of the Molecule-Surface Potential. J. Chem. Phys. 2014, 141, 024701.

(42) Kammler, T.; Lee, J.; Küppers, J. A Kinetic Study of the Interaction of Gaseous H(D) Atoms with $\mathrm{D}(\mathrm{H})$ Adsorbed on $\mathrm{Ni}(100)$ Surfaces. J. Chem. Phys. 1997, 106, 7362-7371.

(43) Kim, J. Y.; Lee, J. Spatial and Kinetic Separation of Eley-Rideal Plus Primary Hot Atom and Secondary Hot Atom Mechanisms in H Atom Abstraction of Adsorbed D Atoms on Pt(111). Phys. Rev. Lett. 1999, 82, 1325-1328.

(44) Kim, J. Y.; Lee, J. Kinetics, Mechanism, and Dynamics of the Gas-phase H(D) Atom Reaction with Adsorbed $\mathrm{D}(\mathrm{H})$ Atom on $\mathrm{Pt}(111)$. J. Chem. Phys. 2000, 113, 28562865 .

(45) Rettner, C. T.; Auerbach, D. J. Dynamics of the Eley-Rideal Reaction of D Atoms with H Atoms Adsorbed on $\mathrm{Cu}(111)$ : Vibrational and Rotational State Distributions of the HD Product. Phys. Rev. Lett. 1995, 74, 4551-4554. 
(46) Rettner, C. T. Reaction of an H Atom Beam with $\mathrm{Cl} / \mathrm{Au}(111)$ : Dynamics of Concurrent Eley-Rideal and Langmuir-Hinshelwood Mechanisms. J. Chem. Phys. 1994, 101, $1529-1546$.

(47) Rettner, C. T.; Auerbach, D. J. Distinguishing the Direct and Indirect Products of a Gas-Surface Reaction. Science 1994, 263, 365-367.

(48) Wehner, S.; Küppers, J. Abstraction of D Adsorbed on Pt (111) Surfaces with Gaseous H Atoms. J. Chem. Phys. 1998, 108, 3353-3359.

(49) Wehner, S.; Küppers, J. Interaction of Gaseous D Atoms with $\mathrm{CH}_{3} \mathrm{I}$ Adsorbed on $\mathrm{Pt}(111), \mathrm{H} / \mathrm{Pt}(111)$, and C/Pt(111) Surfaces: From Hot-Atom to Eley-Rideal Phenomenology. J. Chem. Phys. 1998, 109, 294-300.

(50) Kammler, T.; Küppers, J. Interaction of H Atoms with $\mathrm{Cu(111)} \mathrm{Surfaces:} \mathrm{Adsorption,}$ Absorption, and Abstraction. J. Chem. Phys. 1999, 111, 8115-8123.

(51) Rettner, C. T. Dynamics of the Direct Reaction of Hydrogen Atoms Adsorbed on $\mathrm{Cu}(111)$ with Hydrogen Atoms Incident from the Gas Phase. Phys. Rev. Lett. 1992, 69, 383-386.

(52) Cacciatore, M.; Christoffersen, E.; Rutigliano, M. Adsorption Site and Surface Temperature Effects in CO Formation on $\mathrm{Pt}(111)$ : A New Semiclassical Study. J. Chem. Phys. A 2004, 108, 8810-8818.

(53) Ueta, H.; Gleeson, M. A.; Kleyn, A. W. The Interaction of Hyperthermal Nitrogen with N-covered $\mathrm{Ag}(111)$. J. Chem. Phys. 2011, 135, 074702.

(54) Diekhöner, L.; Hornekær, L.; Mortensen, H.; Jensen, E.; Baurichter, A.; Petrunin, V.; Luntz, A. Indirect Evidence for Strong Nonadiabatic Coupling in $\mathrm{N}_{2}$ Associative Desorption from and Dissociative Adsorption on $\mathrm{Ru}(0001)$. J. Chem. Phys. 2002, 117, $5018-5030$. 
(55) Lykke, K. R.; Kay, B. D. State-to-state Inelastic and Reactive Molecular Beam Scattering from Surfaces. Proc. SPIE 1990, 1208, 18-29.

(56) Kolovos-Vellianitis, D.; Küppers, J. Kinetics of Abstraction of D and $\mathrm{O}$ on $\mathrm{Cu}(110)$ Surfaces by Gaseous H Atoms. J. Phys. Chem. B 2003, 107, 2559-2564.

(57) Krenn, G.; Eibl, C.; Mauritsch, W.; Hebenstreit, E.; Varga, P.; Winkler, A. Adsorption Kinetics and Energetics of Atomic Hydrogen (Deuterium) on Oxygen and Carbon Covered V(100). Surf. Sci. 2000, 445, 343-357.

(58) Kori, M.; Halpern, B. L. Vibrational Energy Distribution of CO in the Oxidation of C on Pt. Chem. Phys. Lett. 1983, 98, 32-36.

(59) Langmuir, I. Part II.- "Heterogeneous Reactions". Chemical Reactions on Surfaces. Trans. Faraday Soc. 1922, 17, 607-620.

(60) Eley, D. D.; Rideal, E. K. Parahydrogen Conversion on Tungsten. Nature 1940, 146, 401-402.

(61) Harris, J.; Kasemo, B. On Precursor Mechanisms for Surface Reactions. Surf. Sci. 1981, 105, L281-L287.

(62) Galparsoro, O.; Pétuya, R.; Busnengo, F.; Juaristi, J. I.; Crespos, C.; Alducin, M.; Larrégaray, P. Hydrogen Abstraction from Metal Surfaces: When Electron-Hole Pair Excitations Strongly Affect Hot-Atom Recombination. Phys. Chem. Chem. Phys. 2016, 18, 31378-31383.

(63) Shimokawa, S.; Namiki, A.; N. Gamo, M.; Ando, T. Temperature Dependence of Atomic Hydrogen-Induced Surface Processes on Ge(100): Thermal Desorption, Abstraction, and Collision-Induced Desorption. J. Chem. Phys. 2000, 113, 6916-6925.

(64) Rutigliano, M.; Cacciatore, M. Isotope and Surface Temperature Effects for Hydrogen Recombination on a Graphite Surface. Chem. Phys. Chem. 2008, 9, 171-181. 
(65) Hansen, B. F.; Billing, G. D. Hydrogen and Deuterium Recombination Rates on a Copper Surface. Surf. Sci. 1997, 373, L333 - L338.

(66) Galparsoro, O.; Pétuya, R.; Juaristi, J. I.; Crespos, C.; Alducin, M.; Larrégaray, P. Energy Dissipation to Tungsten Surfaces upon Eley-Rideal Recombination of $\mathrm{N}_{2}$ and $\mathrm{H}_{2}$. J. Phys. Chem. C 2015, 119, 15434-15442.

(67) Jackson, B.; Persson, M. Effects of Isotopic Substitution on Eley-Rideal Reactions and Adsorbate-mediated Trapping. J. Chem. Phys. 1995, 103, 6257-6269.

(68) Jackson, B.; Persson, M. A Quantum Mechanical Study of Recombinative Desorption of Atomic Hydrogen on a Metal Surface. J. Chem. Phys. 1992, 96, 2378-2386.

(69) Pétuya, R.; Larrégaray, P.; Crespos, C.; Aurel, P.; Busnengo, H. F.; Martínez, A. E. Isotope Effects in Eley-Rideal and Hot-Atom Abstraction Dynamics of Hydrogen from Tungsten (100) and (110) Surfaces. J. Phys. Chem. C 2015, 119, 15325-15332.

(70) Persson, M.; Jackson, B. Flat Surface Study of the Eley-Rideal Dynamics of Recombinative Desorption of Hydrogen on a Metal Surface. J. Chem. Phys. 1995, 102, 1078-1093.

(71) Wheeler, M. C.; Reeves, C. T.; Seets, D. C.; Mullins, C. B. Experimental Study of CO Oxidation by an Atomic Oxygen Beam on $\operatorname{Pt}(111), \operatorname{Ir}(111)$, and $\operatorname{Ru}(001)$. J. Chem. Phys. 1998, 108, 3057-3063.

(72) Zaharia, T.; Kleyn, A. W.; Gleeson, M. A. Eley-Rideal Reactions with N Atoms at $\mathrm{Ru}(0001)$ : Formation of $\mathrm{NO}$ and $\mathrm{N}_{2}$. PRL 2014, 113, 053201.

(73) Markelj, S.; Čadež, I. Production of Vibrationally Excited Hydrogen Molecules by Atom Recombination on $\mathrm{Cu}$ and W Materials. J. Chem. Phys. 2011, 134, 124707. 
(74) Eenshuistra, P. J.; Bonnie, J. H. M.; Los, J.; Hopman, H. J. Observation of Exceptionally High Vibrational Excitation of Hydrogen Molecules Formed by Wall Recombination. Phys. Rev. Lett. 1988, 60, 341-344.

(75) Rideal, E. K. A Note on a Simple Molecular Mechanism for Heterogeneous Catalytic Reactions. Math. Proc. Cambridge. 1939; pp 130-132.

(76) Eley, D. D. The Interchange of Hydrogen in the Adsorbed Film on Tungsten. P. Roy. Soc. A-Math. Phy. 1941; pp 452-464.

(77) Schermann, C.; Pichou, F.; Landau, M.; Čadež, I.; Hall, R. I. Highly Excited Hydrogen Molecules Desorbed from a Surface: Experimental Results. J. Chem. Phys. 1994, 101, $8152-8158$.

(78) Hall, R. I.; Čadež, I.; Landau, M.; Pichou, F.; Schermann, C. Vibrational Excitation of Hydrogen via Recombinative Desorption of Atomic Hydrogen Gas on a Metal Surface. Phys. Rev. Lett. 1988, 60, 337-340.

(79) Pétuya, R.; Crespos, C.; Quintas-Sánchez, E.; Larrégaray, P. Comparative Theoretical Study of $\mathrm{H}_{2}$ Eley-Rideal Recombination Dynamics on W(100) and W(110). J. Phys. Chem. C 2014, 118, 11704-11710.

(80) Halpern, B.; Rosner, D. E. Chemical Energy Accommodation at Catalyst Surfaces. Flow Reactor Studies of the Association of Nitrogen Atoms on Metals at High Temperatures. J. Chem. Soc., Faraday Trans. 1 1978, 74, 1883-1912.

(81) Martin-Gondre, L.; Crespos, C.; Larrégaray, P.; Rayez, J.; van Ootegem, B.; Conte, D. Is the LEPS Potential Accurate Enough to Investigate the Dissociation of Diatomic Molecules on surfaces? Chem. Phys. Lett. 2009, 471, 136-142.

(82) Martin-Gondre, L.; Crespos, C.; Larrégaray, P.; Rayez, J. C.; van Ootegem, B.; Conte, D. Dynamics Simulation of $\mathrm{N}_{2}$ Scattering onto W $(100,110)$ Surfaces: A Strin- 
gent Test for the Recently Developed Flexible Periodic London-Eyring-Polanyi-Sato Potential Energy Surface. J. Chem. Phys. 2010, 132, 204501.

(83) Martin-Gondre, L.; Crespos, C.; Larrégaray, P.; Rayez, J.; Conte, D.; van Ootegem, B. Detailed Description of the Flexible Periodic London-Eyring-Polanyi-Sato Potential Energy Function. Chem. Phys. 2010, 367, 136-147.

(84) Perdew, J. P.; Burke, K.; Ernzerhof, M. Generalized gradient approximation made simple. Phys. Rev. Lett. 1996, 77, 3865-3868.

(85) Busnengo, H. F.; Martínez, A. E. $\mathrm{H}_{2}$ Chemisorption on W(100) and W(110) Surfaces. J. Phys. Chem. C 2008, 112, 5579-5588.

(86) Volpilhac, G.; Salin, A. Dissociative Adsorption of $\mathrm{N}_{2}$ on the W(100) Surface. Surf. Sci. 2004, 556, 129-144.

(87) Bocan, G. A.; Díez Muiño, R.; Alducin, M.; Busnengo, H. F.; Salin, A. The Role of Exchange-correlation Functionals in the Potential Energy Surface and Dynamics of $\mathrm{N}_{2}$ Dissociation on W Surfaces. J. Chem. Phys. 2008, 128, 154704.

(88) Alducin, M.; Díez Muiño, R.; Busnengo, H. F.; Salin, A. Why $\mathrm{N}_{2}$ Molecules with Thermal Energy Abundantly Dissociate on W(100) and Not on W(110). Phys. Rev. Lett. 2006, 97, 056102.

(89) Alducin, M.; Díez Muiño, R.; Busnengo, H. F.; Salin, A. Low Sticking Probability in the Nonactivated Dissociation of $\mathrm{N}_{2}$ Molecules on W(110). J. Chem. Phys. 2006, 125, 144705 .

(90) Martin-Gondre, L.; Bocan, G. A.; Alducin, M.; Juaristi, J. I.; Díez Muiño, R. Energy Dissipation Channels in the Adsorption of $\mathrm{N}$ on $\mathrm{Ag}(111)$. Comp. Theor. Chem. 2012, 990, 126-131. 
(91) Adelman, S. A. Generalized Langevin Theory for Many-body Problems in Chemical Dynamics: General Formulation and the Equivalent Harmonic Chain Representation. J. Chem. Phys. 1979, 71, 4471-4486.

(92) Tully, J. C. Dynamics of Gas-Surface Interactions: 3D Generalized Langevin Model Applied to fcc and bcc Surfaces. J. Chem. Phys. 1980, 73, 1975-1985.

(93) Polanyi, J. C.; Wolf, R. J. Dynamics of Simple Gas-Surface Interaction. II. Rotationally Inelastic Collisions at Rigid and Moving Surfaces. J. Chem. Phys. 1985, 82, 15551566.

(94) Dohle, M.; Saalfrank, P.; Uzer, T. The Dissociation of Diatomic Molecules on Vibrating Surfaces: A Semiclassical Generalized Langevin Approach. J. Chem. Phys. 1998, 108, $4226-4236$.

(95) Busnengo, H. F.; Dong, W.; Salin, A. Trapping, Molecular Adsorption, and Precursors for Nonactivated Chemisorption. Phys. Rev. Lett. 2004, 93, 236103.

(96) Nattino, F.; Galparsoro, O.; Costanzo, F.; Díez Muiño, R.; Alducin, M.; Kroes, G.-J. Modeling Surface Motion Effects in $\mathrm{N}_{2}$ Dissociation on W (110): Ab Initio Molecular Dynamics Calculations and Generalized Langevin Oscillator Model. J. Chem. Phys. 2016, $144,244708$.

(97) Juaristi, J. I.; Alducin, M.; Díez Muiño, R.; Busnengo, H. F.; Salin, A. Role of Electron-Hole Pair Excitations in the Dissociative Adsorption of Diatomic Molecules on Metal Surfaces. Phys. Rev. Lett. 2008, 100, 116102.

(98) Juaristi, J.; Díaz, E.; Bocan, G. A.; Díez Muiño, R.; Alducin, M.; Blanco-Rey, M. Angular Distributions and Rovibrational Excitation of $\mathrm{N}_{2}$ Molecules Recombined on N-covered $\operatorname{Ag}(111)$ by the Eley-Rideal Mechanism. Catal. Today 2015, 244, 115-121. 
(99) Rettner, C. T.; Auerbach, D. J. Dynamics of the Formation of HD from D(H) Atoms Colliding with $\mathrm{H}(\mathrm{D}) / \mathrm{Cu}$ (111): a Model Study of an Eley-Rideal Reaction. Surf. Sci. 1996, 357, 602-608.

(100) Rettner, C.; Auerbach, D.; Tully, J.; Kleyn, A. Chemical dynamics at the gas-surface interface. J. Phys. Chem. 1996, 100, 13021-13033. 


\section{Graphical TOC Entry}

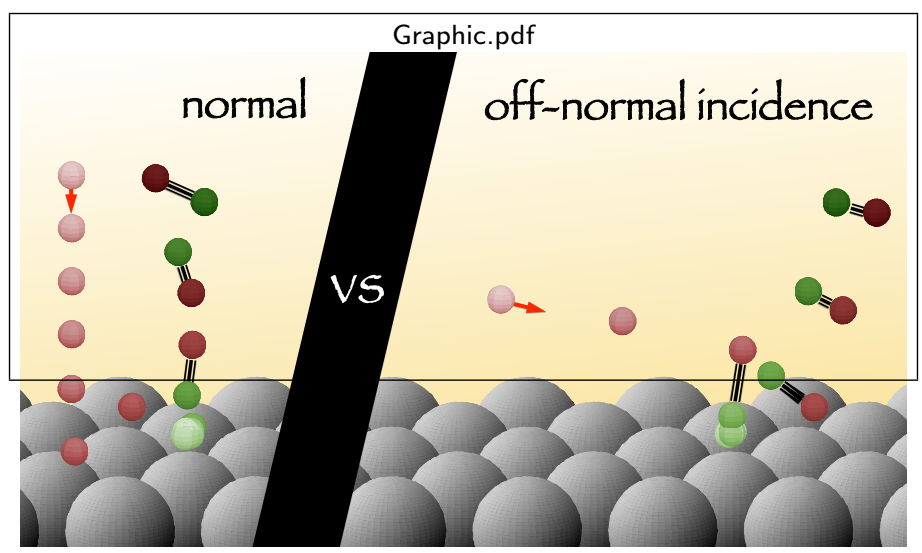

